Supporting Information

\title{
2,3-Naphtho-fused BODIPYs as near-infrared absorbing dyes
}

\author{
Sho Yamazawa, Mika Nakashima, Yukie Suda, Ryuhei Nishiyabu, and Yuji Kubo* \\ Department of Applied Chemistry, Graduate School of Urban Environmental Sciences, Tokyo \\ Metropolitan University, 1-1 Minami-ohsawa, Hachioji, Tokyo 192-0397, Japan \\ E-mail: yujik@tmu.ac.jp
}

\section{Table of Contents}

Figure S1. (a) X ray crystal structure of packing dimer $1 \mathbf{a}$.

p. SI-2

Figure S2. Variable-temperature ${ }^{1} \mathrm{H}$ NMR $(500 \mathrm{MHz})$ spectra of $\mathbf{1 b}$.

p. SI-2

Figure S3. ${ }^{19} \mathrm{~F}$ NMR $(470 \mathrm{MHz})$ spectra of $\mathbf{1 b}$.

p. SI-3

Figure S4. Solvent effect on the absorption and fluorescence spectra of 1a.

p. SI-3

Figure S5. Solvent effect on the absorption and fluorescence spectra of $\mathbf{1 b}$.

p. SI-3

Figure S6. Solvent effect on the absorption and fluorescence spectra of $\mathbf{2}$.

p. SI-4

Table S1. Absorption and fluorescence spectra of $\mathbf{1 b}$ and $\mathbf{2}$ in various solvents

p. SI-4

Table S2. Theoretical data of the dyes.

p. SI-4

Figure S7. Cyclic voltammograms of dyes 7, 1a and $\mathbf{1 b}$.

p. SI-5

Table S3. The $\lambda_{\max }$ and HOMO and LUMO energies of dyes 7, 1a and $\mathbf{1 b}$.

p. SI-5

Figure S8. ${ }^{1} \mathrm{H}$ NMR spectrum of $\mathbf{5 a}$.

p. SI-6

Figure S9. ${ }^{13} \mathrm{C}$ NMR spectrum of $\mathbf{5 a}$.

p. SI-7

Figure S10. High-resolution FAB mass spectrum of $\mathbf{5 a}$.

p. SI-8

Figure S11. ${ }^{1} \mathrm{H}$ NMR spectrum of $\mathbf{5 b}$.

p. SI-9

Figure S12. ${ }^{13} \mathrm{C}$ NMR spectrum of $5 \mathbf{b}$.

p. SI-10

Figure S13. High-resolution FAB mass spectrum of $\mathbf{5 b}$.

p. SI-11

Figure S14. ${ }^{1} \mathrm{H}$ NMR spectrum of 6 a.

p. SI-12

Figure S15. High-resolution APCI mass spectrum of $6 \mathbf{a}$.

p. SI-13

Figure S16. ${ }^{1} \mathrm{H}$ NMR spectrum of $\mathbf{1 a}$.

p. SI-14

Figure S17. ${ }^{13} \mathrm{C}$ NMR spectrum of $\mathbf{1 a}$.

p. SI-15

Figure S18. FAB mass spectrum of $\mathbf{1 a}$.

p. SI-16

Figure S19. ${ }^{1} \mathrm{H}$ NMR spectrum of $\mathbf{1 b}$.

p. SI-17

Figure S20. ${ }^{13} \mathrm{C}$ NMR spectrum of $\mathbf{1 b}$.

p. SI-18

Figure S21. FAB mass spectrum of $\mathbf{1 b}$.

p. SI-19

Figure S22. ${ }^{1} \mathrm{H}$ NMR spectrum of 2.

p. SI-20

Figure S23. High-resolution APCI mass spectrum of 2.

p. SI-21

Single crystal X-ray diffraction study

p. SI-22

Figure S24. (a) Atom numbering Scheme of 1a and (b) its bond lengths and bond angles.

p. SI-27

Table S4. Atom coordinates and absolute energy levels for 7.

p. SI-32

Table S5. Atom coordinates and absolute energy levels for $\mathbf{1 a}$.

p. SI-33

Table S6. Atom coordinates and absolute energy levels for $\mathbf{1 b}$.

p. SI-34

Table S7. Atom coordinates and absolute energy levels for $\mathbf{2}$.

p. SI-35 
(a)

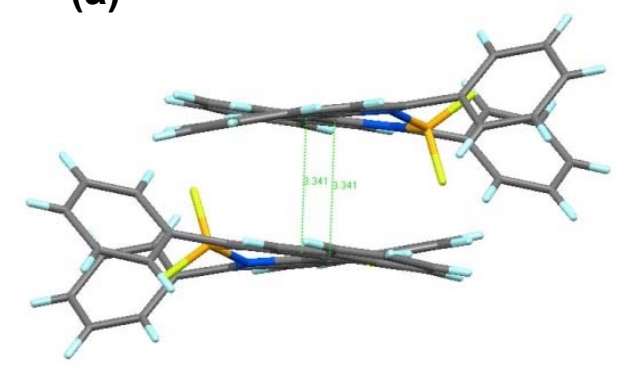

(b)

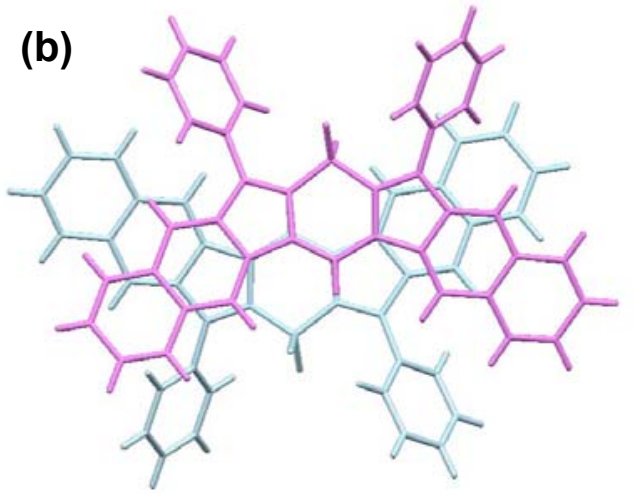

Figure S1. (a) X ray crystal structure of packing dimer 1a, (b) Its front view.
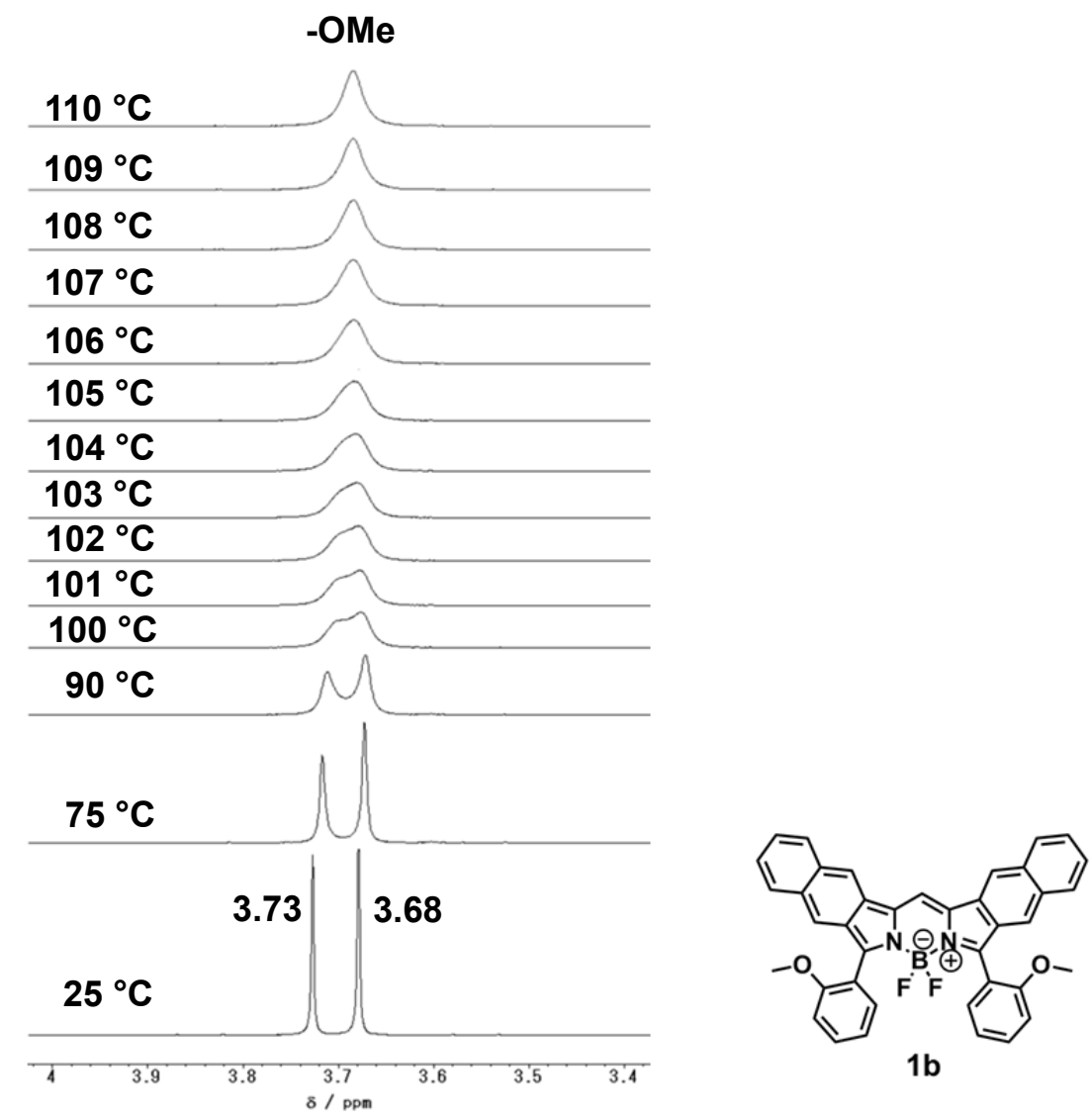

Figure S2. Variable-temperature ${ }^{1} \mathrm{H}$ NMR $(500 \mathrm{MHz})$ spectra of $\mathbf{1 b}$ in DMSO- $d_{6} .[\mathbf{1 b}]=5 \mathrm{mM}$. 


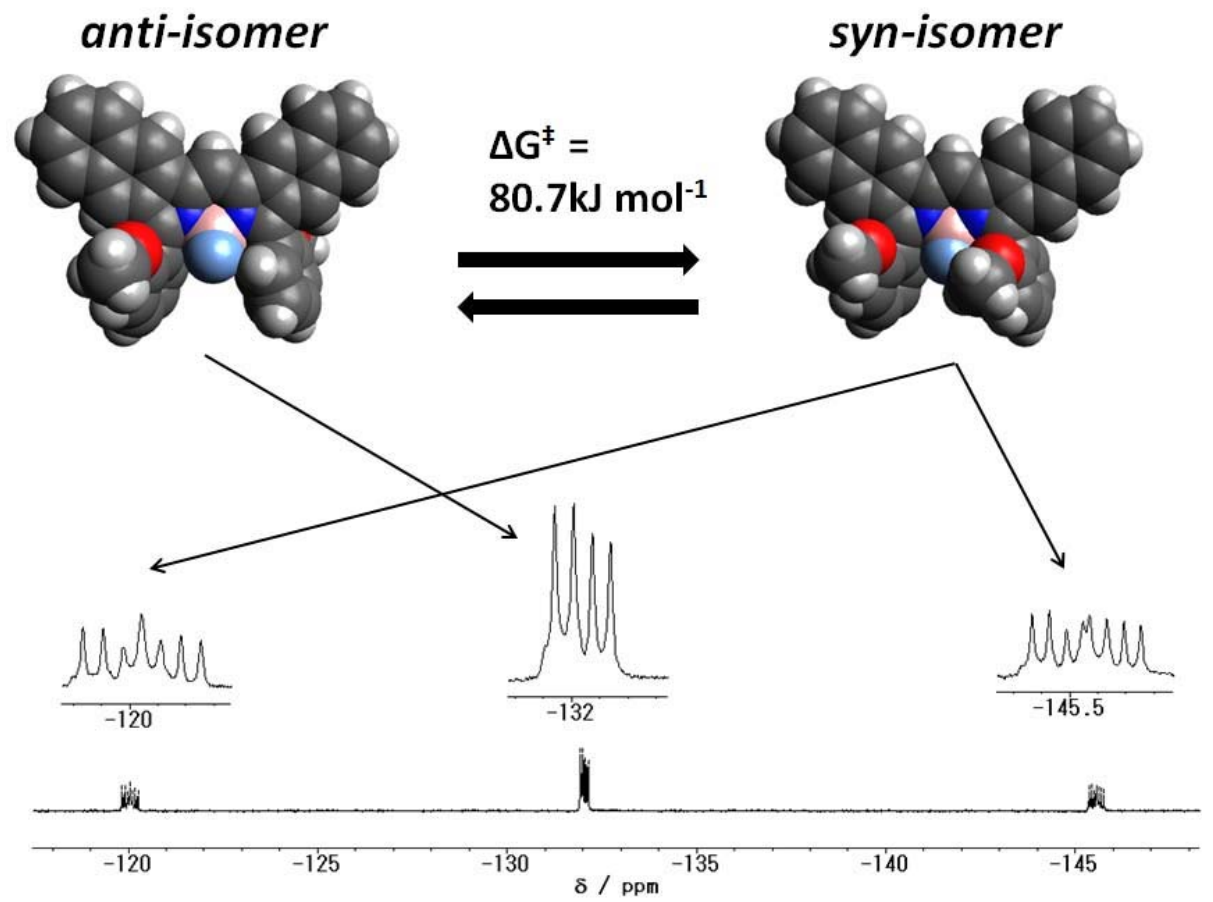

Figure S3. ${ }^{19} \mathrm{~F}$ NMR $(470 \mathrm{MHz})$ spectra of $\mathbf{1 b}$ in DMSO- $d_{6}$ at $25^{\circ} \mathrm{C}$. $[\mathbf{1 b}]=5 \mathrm{mM}$.

(a)

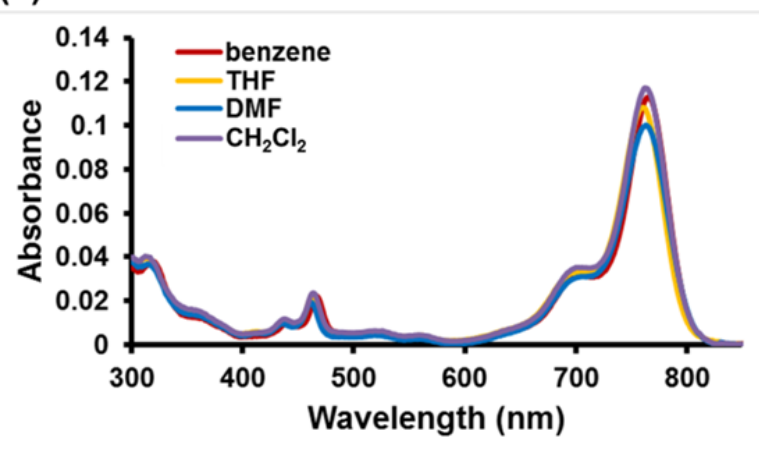

(b)

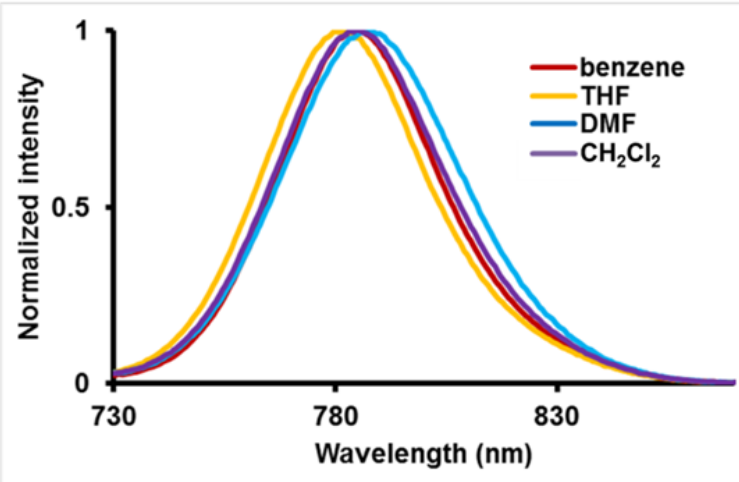

Figure S4. Solvent effect on (a) absorption and (b) fluorescence spectra of $1 \mathrm{a}(1 \mu \mathrm{M})$ at $25^{\circ} \mathrm{C} \cdot \lambda_{\mathrm{ex}}=720 \mathrm{~nm}$

(a)

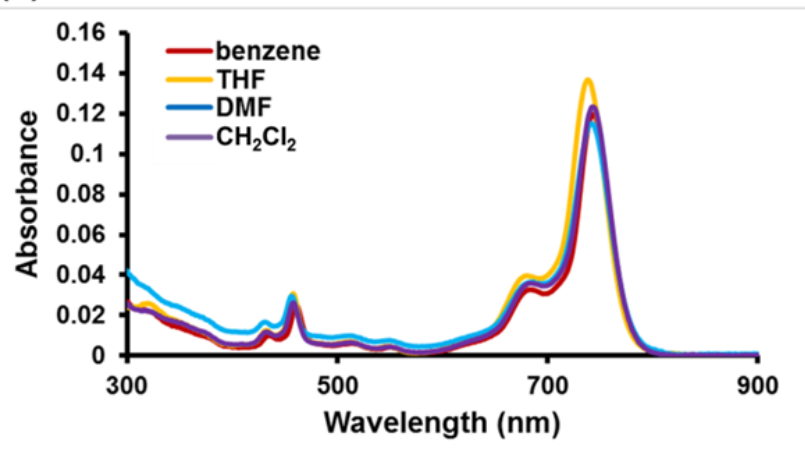

(b)

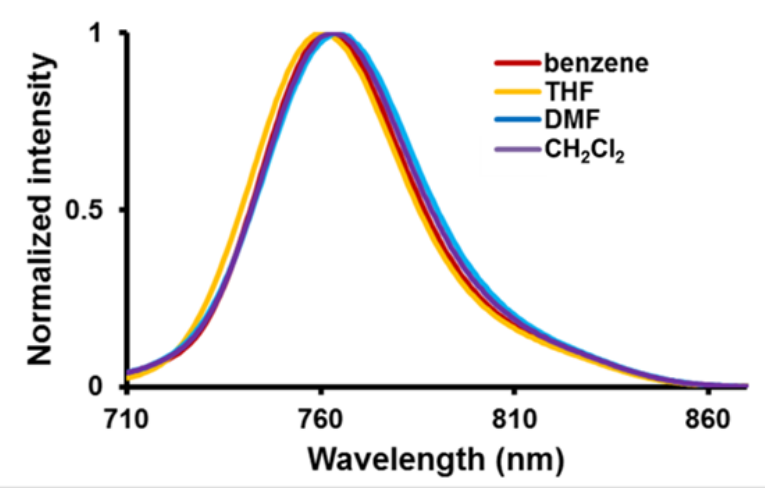

Figure S5. Solvent effect on (a) absorption and (b) fluorescence spectra of $1 \mathbf{b}(1 \mu \mathrm{M})$ at $25^{\circ} \mathrm{C} \cdot \lambda_{\text {ex }}=700 \mathrm{~nm}$. 
(a)

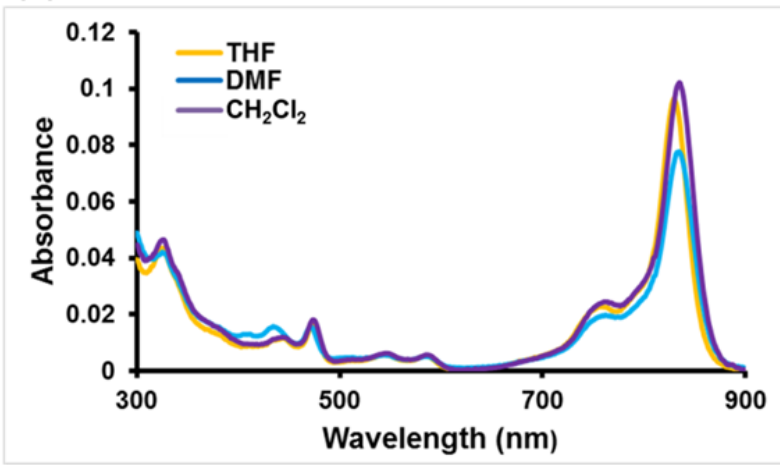

(b)

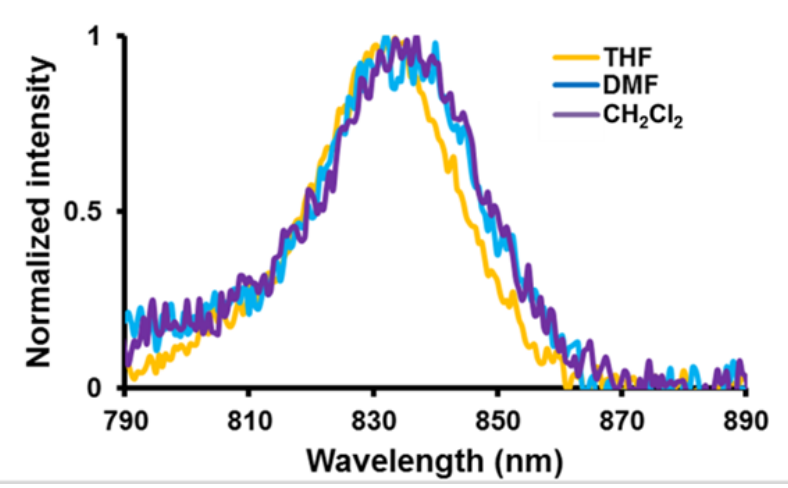

Figure S6. Solvent effect on (a) absorption and (b) fluorescence spectra of $2(1 \mu \mathrm{M})$ at $25^{\circ} \mathrm{C}$. $\lambda_{\mathrm{ex}}=720 \mathrm{~nm}$.

Table S1. Absorption and fluorescent spectra of $\mathbf{1 b}(1 \mu \mathrm{M})$ and $\mathbf{2}(1 \mu \mathrm{M})$ in various solvents at $25^{\circ} \mathrm{C}$.

\begin{tabular}{cccccccc}
\hline Dye & Solvent & $\lambda_{\max }(\mathrm{nm})$ & $\varepsilon^{\mathrm{a}}$ & $\Delta \lambda^{\mathrm{b}}(\mathrm{nm})$ & $\lambda_{\mathrm{em}}{ }^{\mathrm{c}}(\mathrm{nm})$ & $\Delta_{s}{ }^{\mathrm{d}}\left(\mathrm{cm}^{-1}\right)$ & $\Phi_{\mathrm{F}}(\%)$ \\
\hline $\mathbf{1 b}$ & $\mathrm{THF}$ & 738 & 1.37 & 98 & 760 & 392 & 29 \\
& $\mathrm{C}_{6} \mathrm{H}_{6}$ & 743 & 1.20 & 103 & 763 & 353 & 34 \\
& $\mathrm{CH}_{2} \mathrm{Cl}_{2}$ & 743 & 1.23 & 103 & 764 & 370 & 27 \\
& $\mathrm{DMF}$ & 741 & 1.15 & 101 & 764 & 406 & 24 \\
\hline $\mathbf{2}$ & $\mathrm{THF}$ & 830 & 0.962 & 190 & 832 & 29 & 1.3 \\
& $\mathrm{C}_{6} \mathrm{H}_{6}{ }^{\mathrm{e}}$ & - & - & - & - & - & - \\
& $\mathrm{CH}_{2} \mathrm{Cl}_{2}$ & 835 & 1.02 & 195 & 837 & 29 & 1.3 \\
& $\mathrm{DMF}$ & 835 & 0.776 & 195 & 836 & 14 & 0.84 \\
\hline
\end{tabular}

${ }^{\mathrm{a}}$ Molar extinction coefficient, in $10^{5} \mathrm{M}^{-1} \mathrm{~cm}^{-1} \cdot{ }^{\mathrm{b}} \Delta \lambda=\lambda_{\max }-\lambda_{\max }$ of $7 .{ }^{\mathrm{c}} \lambda_{\mathrm{ex}}=720 \mathrm{~nm}$ for 1a and $2 . \lambda_{\mathrm{ex}}=700 \mathrm{~nm}$ for 1b. $\lambda_{\mathrm{ex}}=600 \mathrm{~nm}$ for $7 .{ }^{\mathrm{d}} \Delta_{\mathrm{s}}=\left(1 / \lambda_{\max }\right)-\left(1 / \lambda_{\mathrm{em}}\right) .{ }^{\mathrm{e}}$ Dye 2 has a low solubility in benzene.

Table S2. Theoretical data of the dyes of 1a-b, 2, and 7.

\begin{tabular}{cccccc}
\hline Dye & $\begin{array}{c}\lambda_{\max }(\mathrm{calcd})^{\mathrm{a}} \\
(\mathrm{nm})\end{array}$ & $f(\mathrm{calcd})^{\mathrm{a}}$ & HOMO $(\mathrm{eV})$ & LUMO $(\mathrm{eV})$ & Calculated assignment \\
\hline $\mathbf{7}$ & 541 & 0.7643 & -4.86 & -2.54 & $\begin{array}{r}\text { HOMO } \rightarrow \text { LUMO } \\
(0.712)^{\mathrm{b}}\end{array}$ \\
$\mathbf{1 a}$ & 647 & 0.7062 & -4.52 & -2.53 & $\begin{array}{r}\text { HOMO } \rightarrow \text { LUMO } \\
(0.709)^{\mathrm{b}}\end{array}$ \\
$\mathbf{1 b}$ & 621 & 0.6723 & -4.42 & -2.33 & $\begin{array}{r}\text { HOMO } \rightarrow \text { LUMO } \\
(0.706)^{\mathrm{b}}\end{array}$ \\
$\mathbf{2}$ & 700 & 0.4745 & -4.44 & -2.52 & $\begin{array}{r}\text { HOMO } \rightarrow \text { LUMO } \\
(0.709)^{\mathrm{b}}\end{array}$ \\
\hline
\end{tabular}

${ }^{\mathrm{a}}$ The longest-wavelength absorption bands and oscillator strengths $(f)$ were calculated using B3LYP/6-31G(d,p).

${ }^{\mathrm{b}}$ Configuration interaction coefficient. 


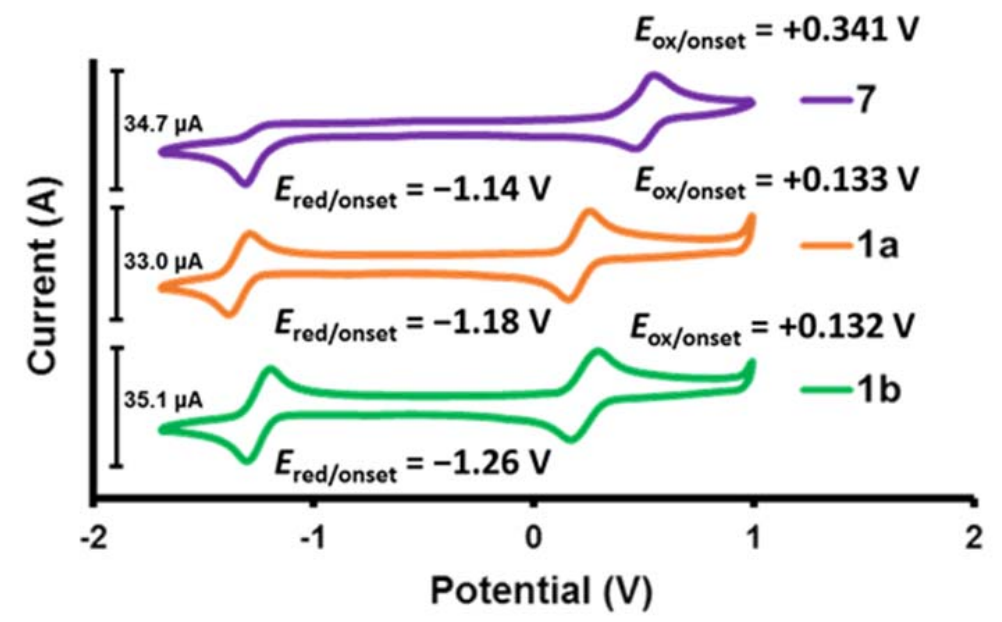

Figure S7. Cyclic voltammograms of dyes 7, $\mathbf{1 a}$ and $\mathbf{1 b}$ measured in a $\mathrm{CH}_{3} \mathrm{CN} / o$-dichlorobenzene $(2: 3 \mathrm{v} / \mathrm{v})$ mixture containing $0.1 \mathrm{M} \mathrm{TBAPF}_{6}$ at room temperature. Ferrocene $(\mathrm{Fc})$ was used as an internal reference.

Table S3. The $\lambda_{\max }$ and HOMO and LUMO energies of dyes $\mathbf{7}$, $\mathbf{1 a}$ and $\mathbf{1} \mathbf{b}$, being calculated from the CV measurements in $\mathrm{CH}_{3} \mathrm{CN} / o$-dichlorobenzene $(2: 3 \mathrm{v} / \mathrm{v})$.

\begin{tabular}{cccc}
\hline Dye & $\lambda_{\max }(\mathrm{nm})^{\mathrm{a}}$ & HOMO $(\mathrm{eV})^{\mathrm{b}}$ & LUMO $(\mathrm{eV})^{\mathrm{c}}$ \\
\hline $\mathbf{7}$ & 640 & -5.03 & -3.55 \\
$\mathbf{1 a}$ & 761 & -4.83 & -3.52 \\
1b & 738 & -4.83 & -3.44
\end{tabular}

${ }^{a}$ in THF. ${ }^{b} \mathrm{E}(\mathrm{HOMO})=-\left(\mathrm{E}_{\mathrm{ox}}-\mathrm{E}_{\mathrm{Fc} / \mathrm{Fc}}+4.8\right) .{ }^{\mathrm{c}} \mathrm{E}(\mathrm{LUMO})=-\left(\mathrm{E}_{\mathrm{red}}-\mathrm{E}_{\mathrm{Fc} / \mathrm{Fc}}+4.8\right)$.

\section{Characterization}




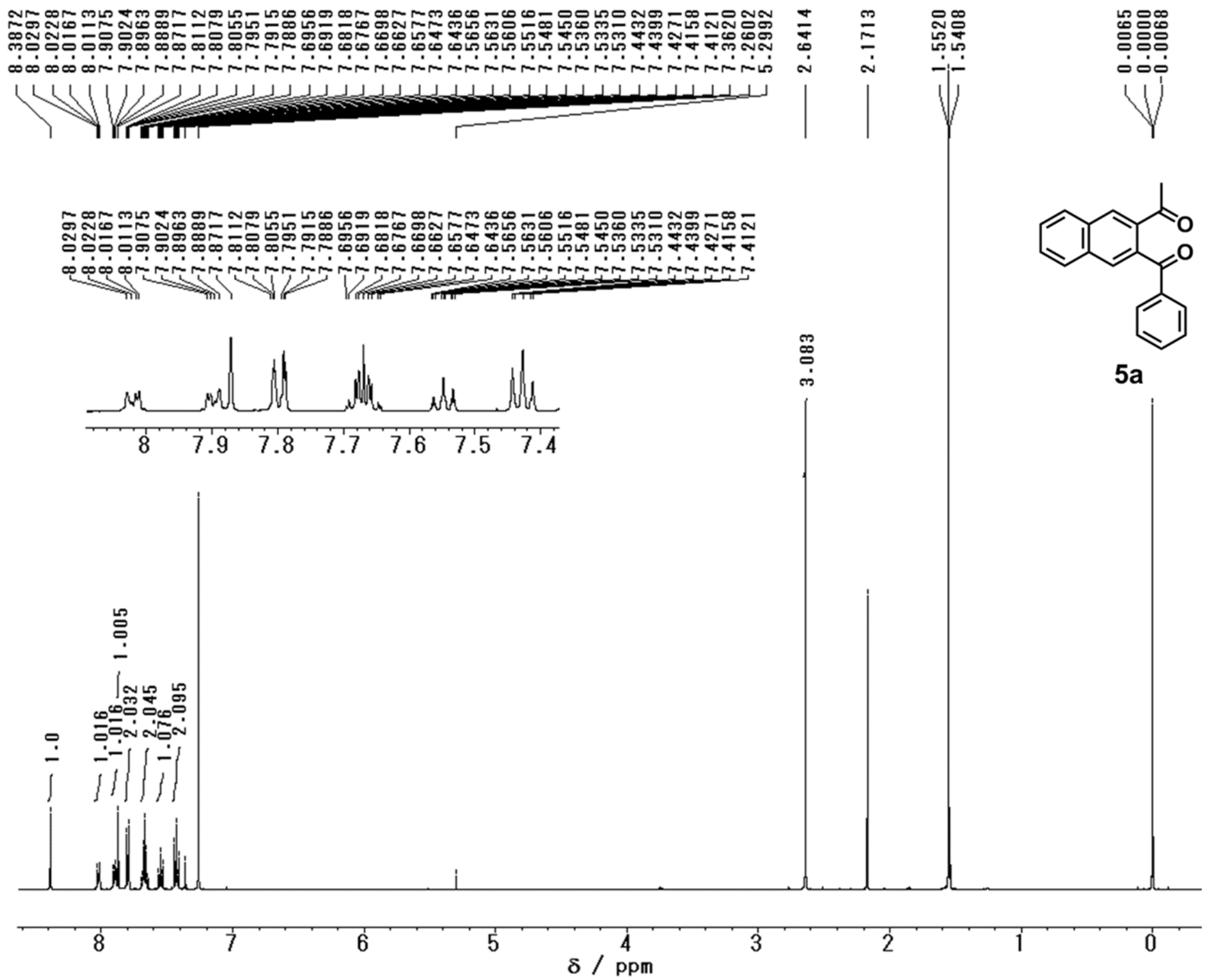

Figure S8. ${ }^{1} \mathrm{H}$ NMR spectrum of $\mathbf{5 a}(500 \mathrm{MHz})$ in $\mathrm{CDCl}_{3}$. 

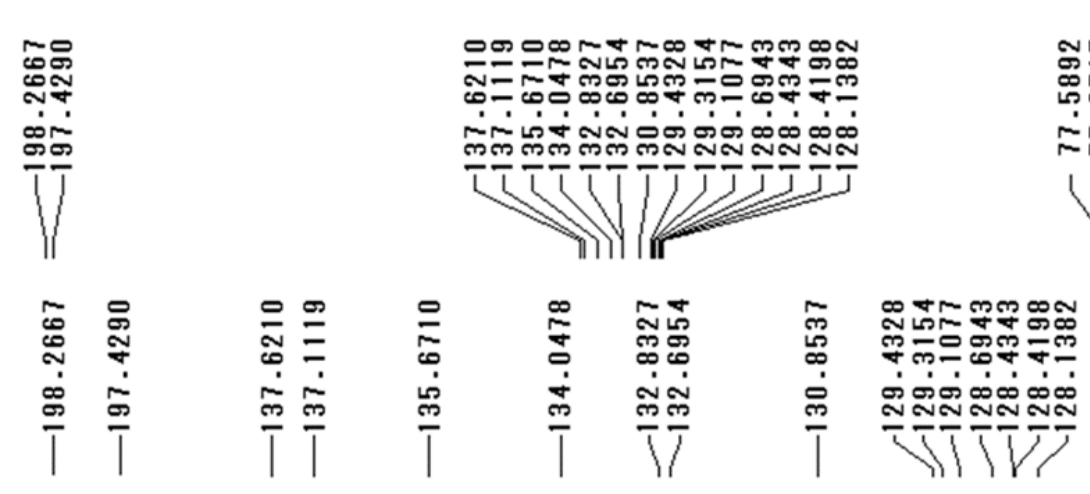

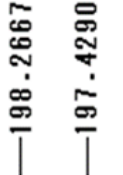

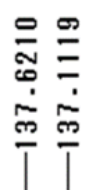

$\underset{\substack{0 \\ 0}}{\substack{0 \\ 0}}$

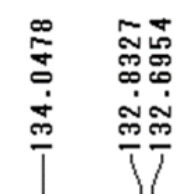

苛

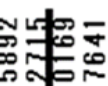

$\sim \pi+\infty$

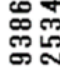

무

음

|

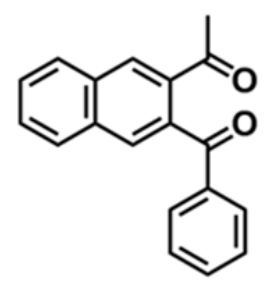

$5 a$
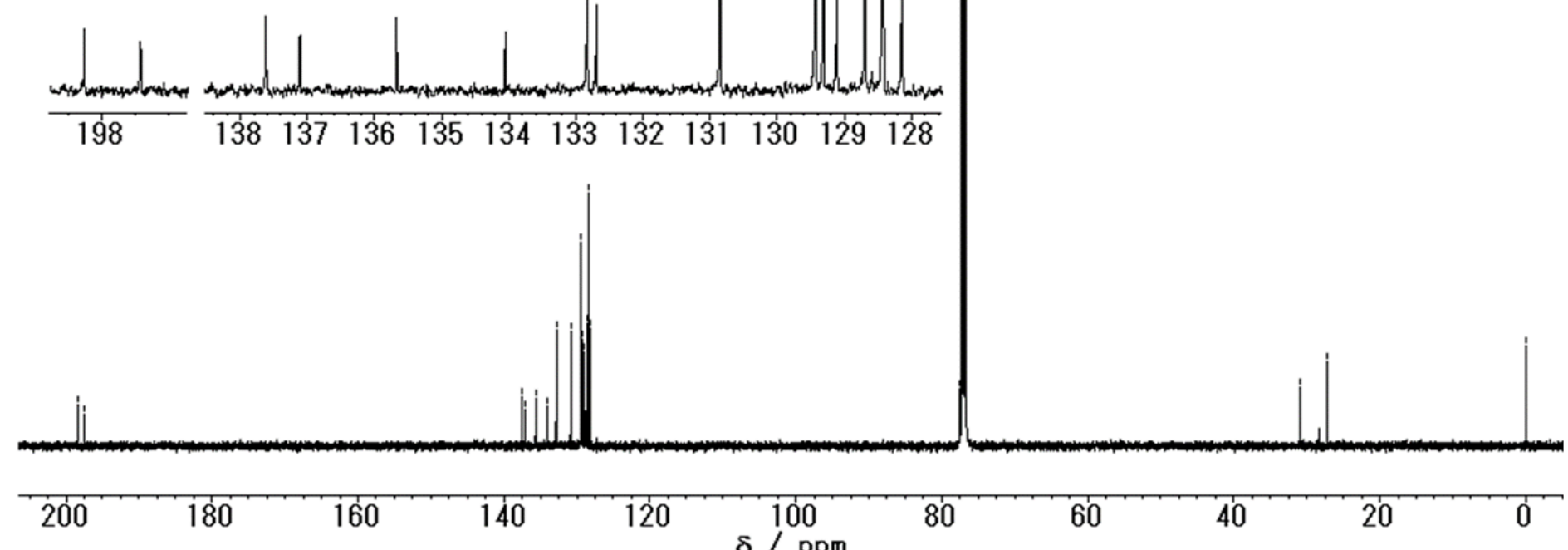

Figure S9. ${ }^{13} \mathrm{C}$ NMR spectrum of $\mathbf{5 a}(125 \mathrm{MHz})$ in $\mathrm{CDCl}_{3}$. 
[ Mass Spectrum ]

Data : 20151128-yamazawaEXP139-2-002

RT : $3.31 \mathrm{~min}$ Scan\# : 19

Element :

Mass Tolerance :

Unsaturation :

Date : 28-Nov-2015 12:41
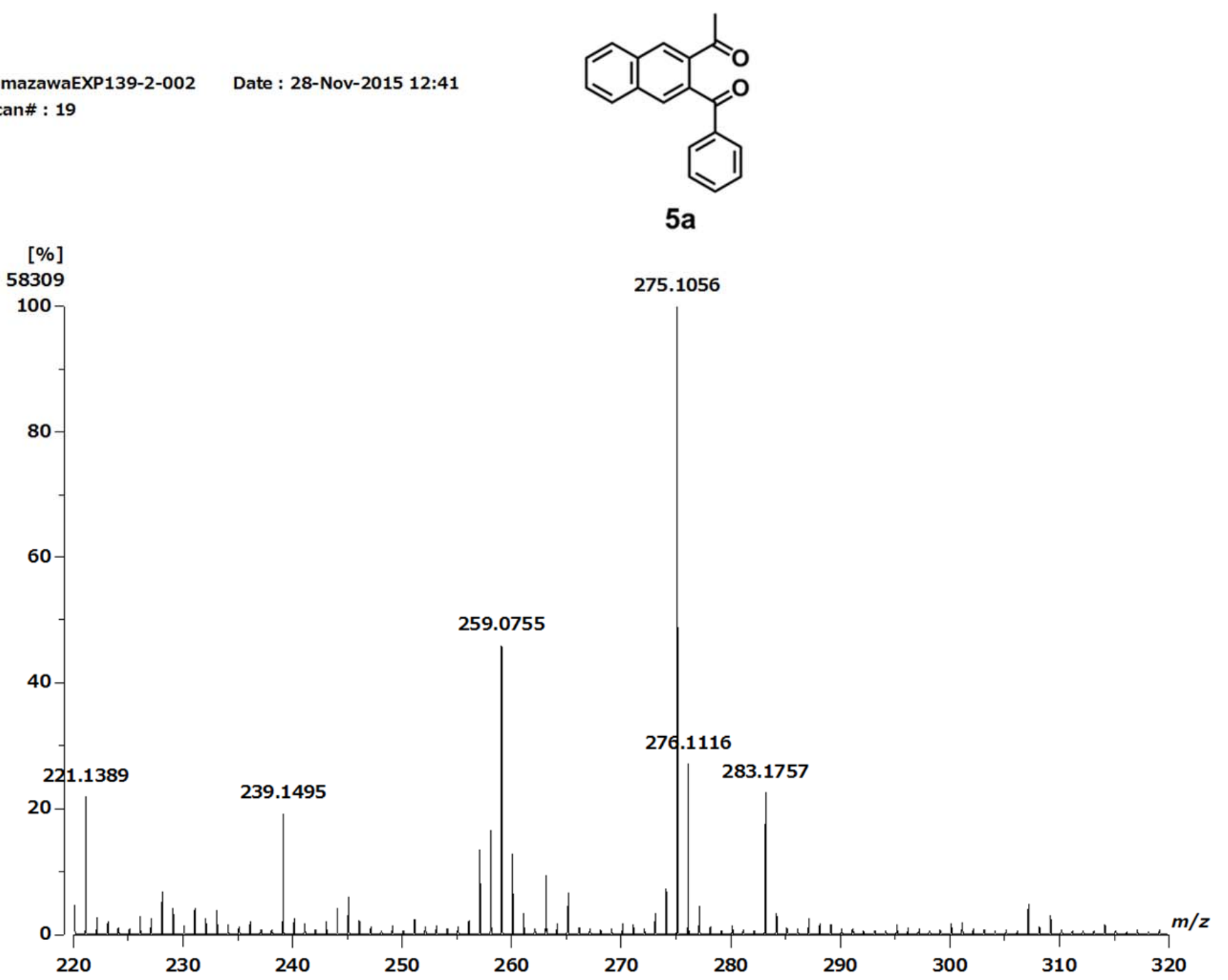

$5 a$

Figure S10. High-resolution FAB mass spectrum of $\mathbf{5 a}$. 


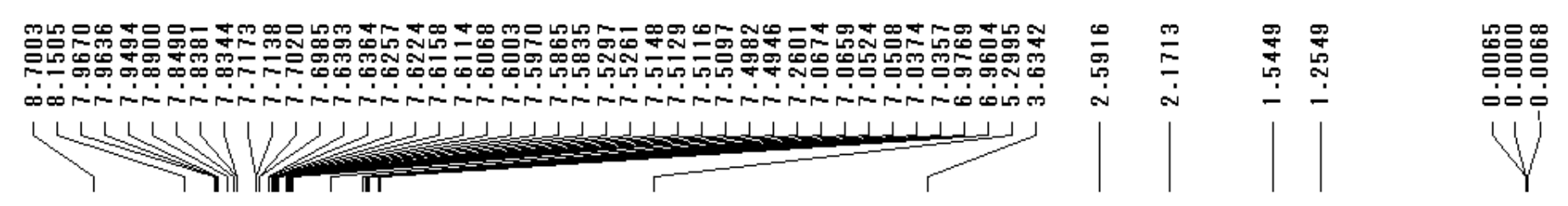

\section{무유.

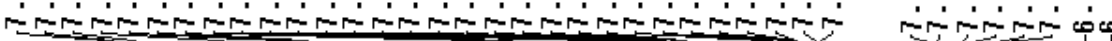
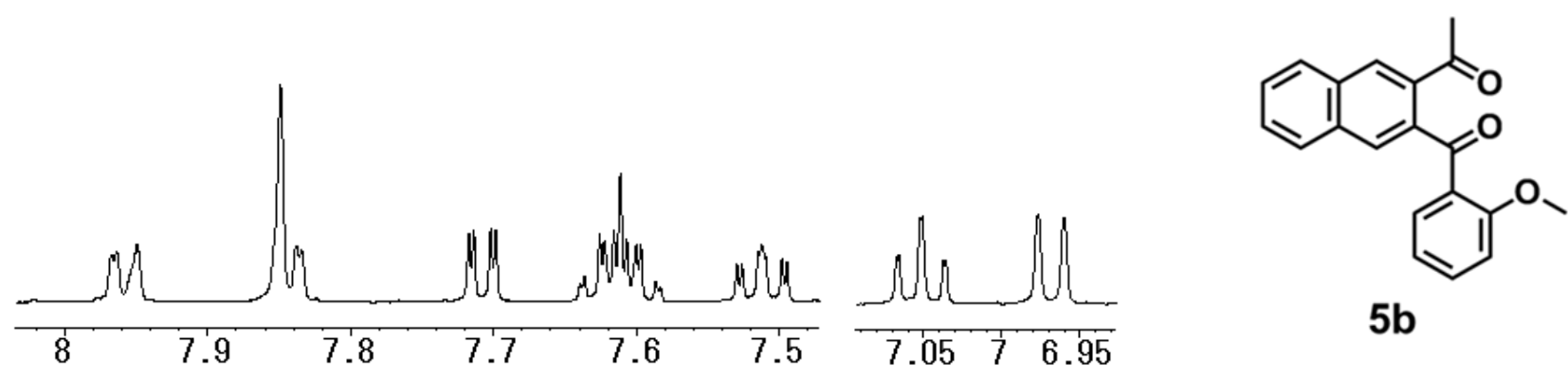

$5 b$

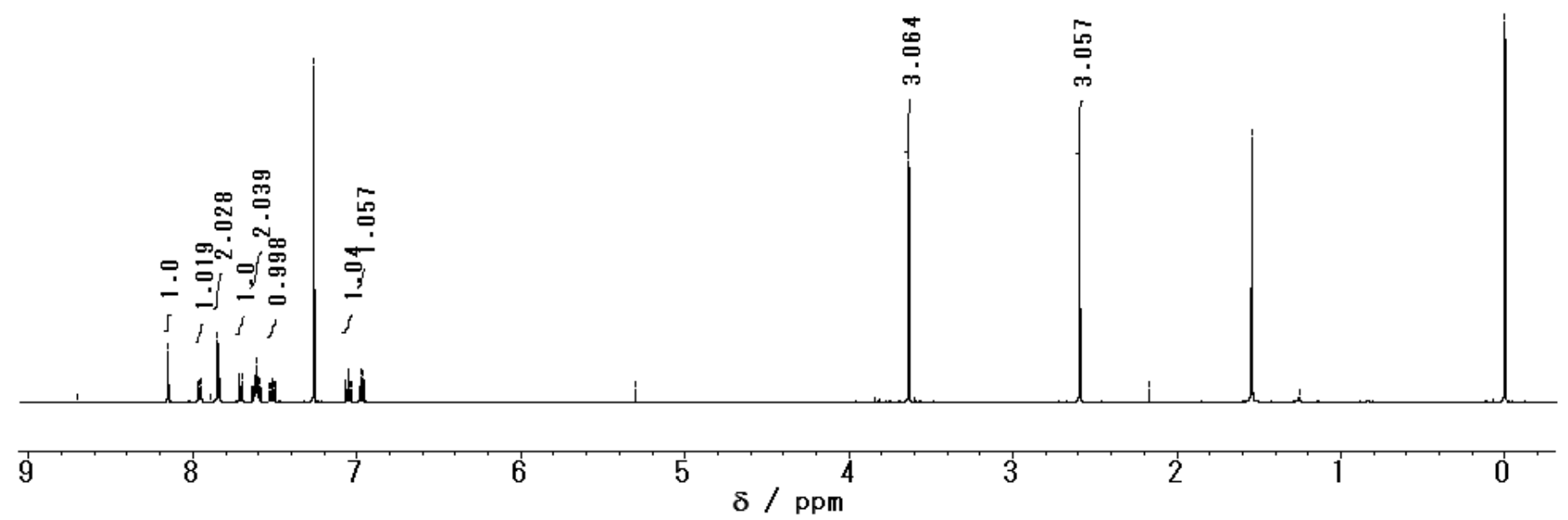

Figure S11. ${ }^{1} \mathrm{H}$ NMR spectrum of $\mathbf{5 b}(500 \mathrm{MHz})$ in $\mathrm{CDCl}_{3}$. 

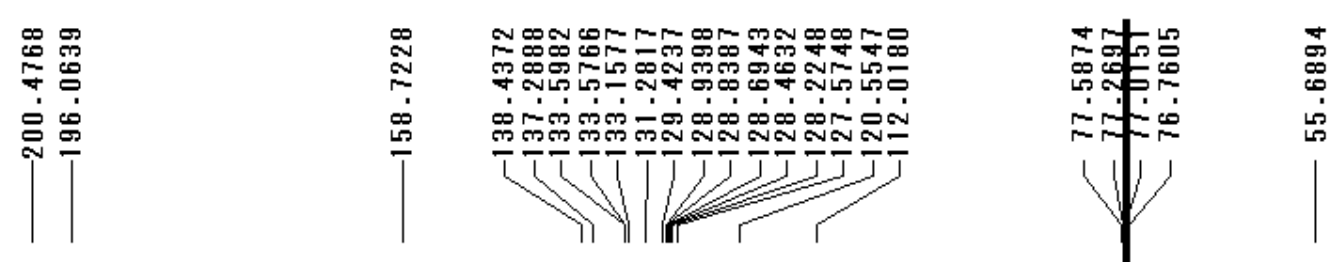

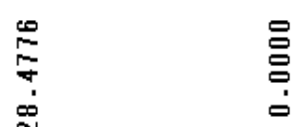

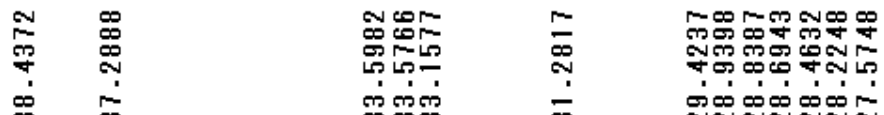

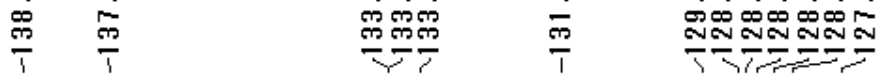
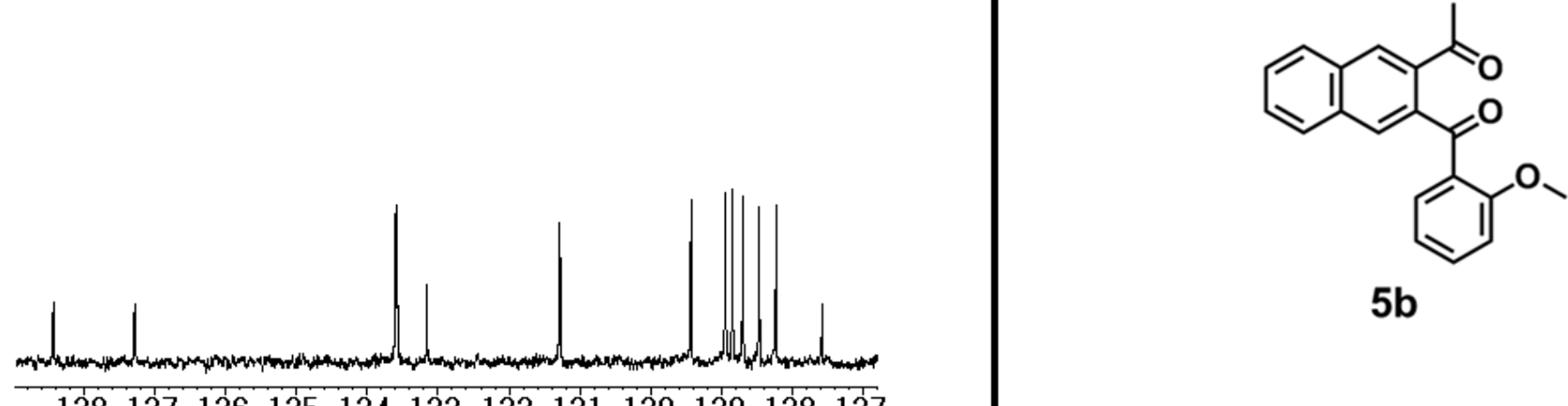

$5 b$

$\begin{array}{lllllllllllll}138 & 137 & 136 & 135 & 134 & 133 & 132 & 131 & 130 & 129 & 128 & 127\end{array}$

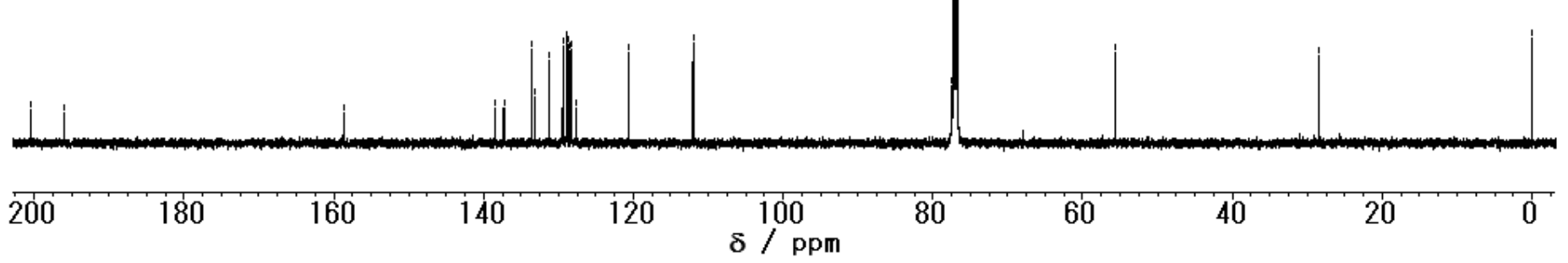

Figure S12. ${ }^{13} \mathrm{C}$ NMR spectrum of $5 \mathbf{b}(125 \mathrm{MHz})$ in $\mathrm{CDCl}_{3}$ 
[ Mass Spectrum ]

Data : 20151128-nakashimaEXP57-3-003

RT : $0.51 \mathrm{~min}$ Scan\# : 4

Element :

Mass Tolerance :

Unsaturation :

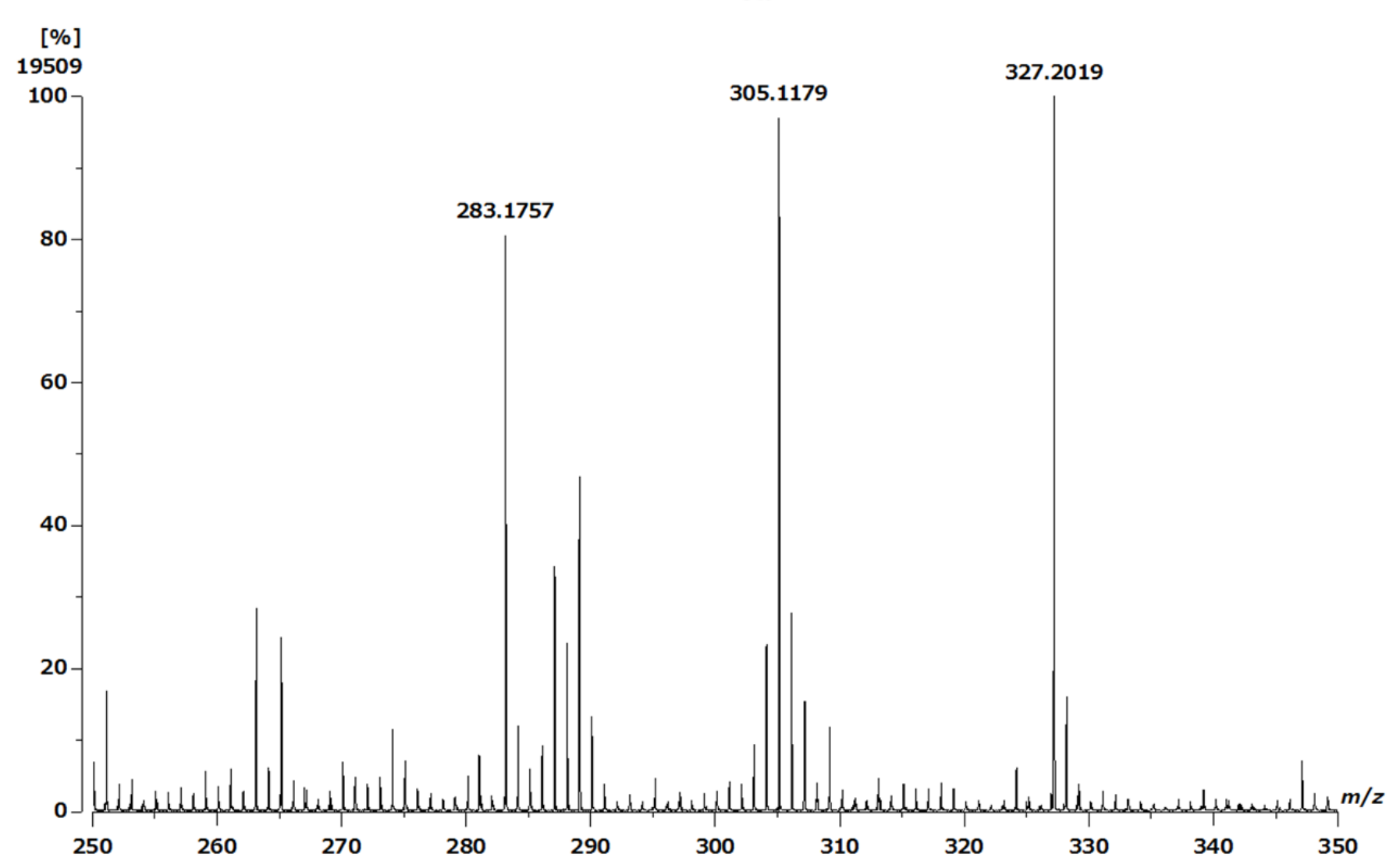

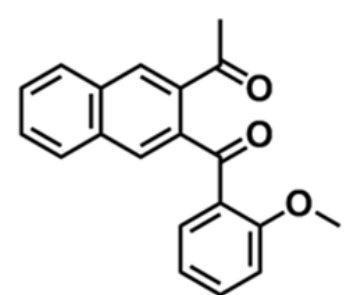

$5 b$

Figure S13. High-resolution FAB mass spectrum of 5b. 


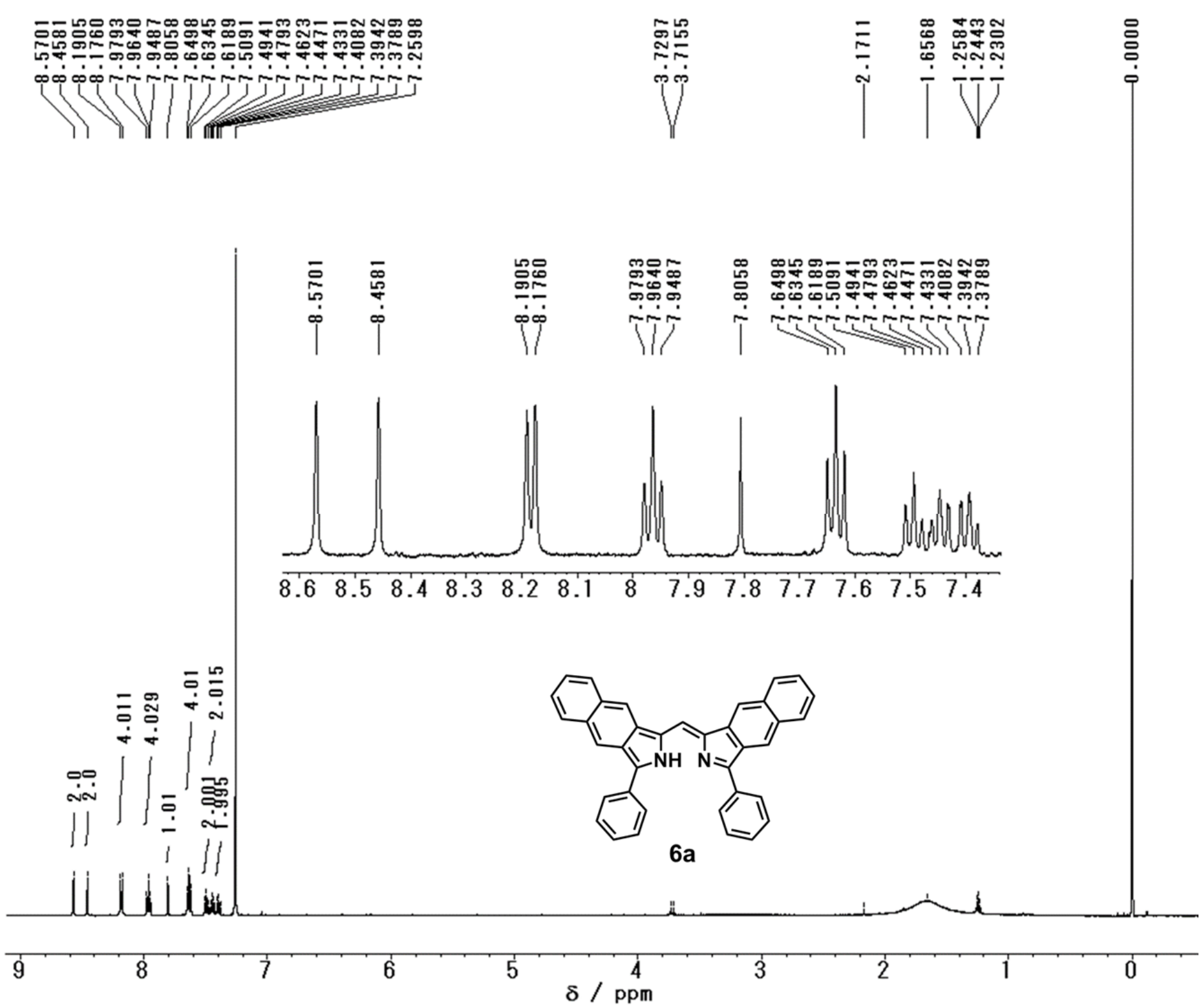

Figure S14. ${ }^{1} \mathrm{H}$ NMR spectrum of $\mathbf{6 a}(500 \mathrm{MHz})$ in $\mathrm{CDCl}_{3}$. 
Generic Display Report

$\begin{array}{llll}\text { Analysis Info } & & \text { Acquisition Date } & \text { 11/27/2015 11:45:34 AM } \\ \text { Analysis Name } & \text { D:IDatalsyn1lakashimalexp55.d } & & \\ \text { Method } & \text { APCl_pos_wide.m } & \text { Operator } & \text { BDAL@DE } \\ \text { Sample Name } & \text { exp55 } & \text { Instrument } & \text { micrOTOF } \\ \text { Comment } & & \text { intiof }\end{array}$
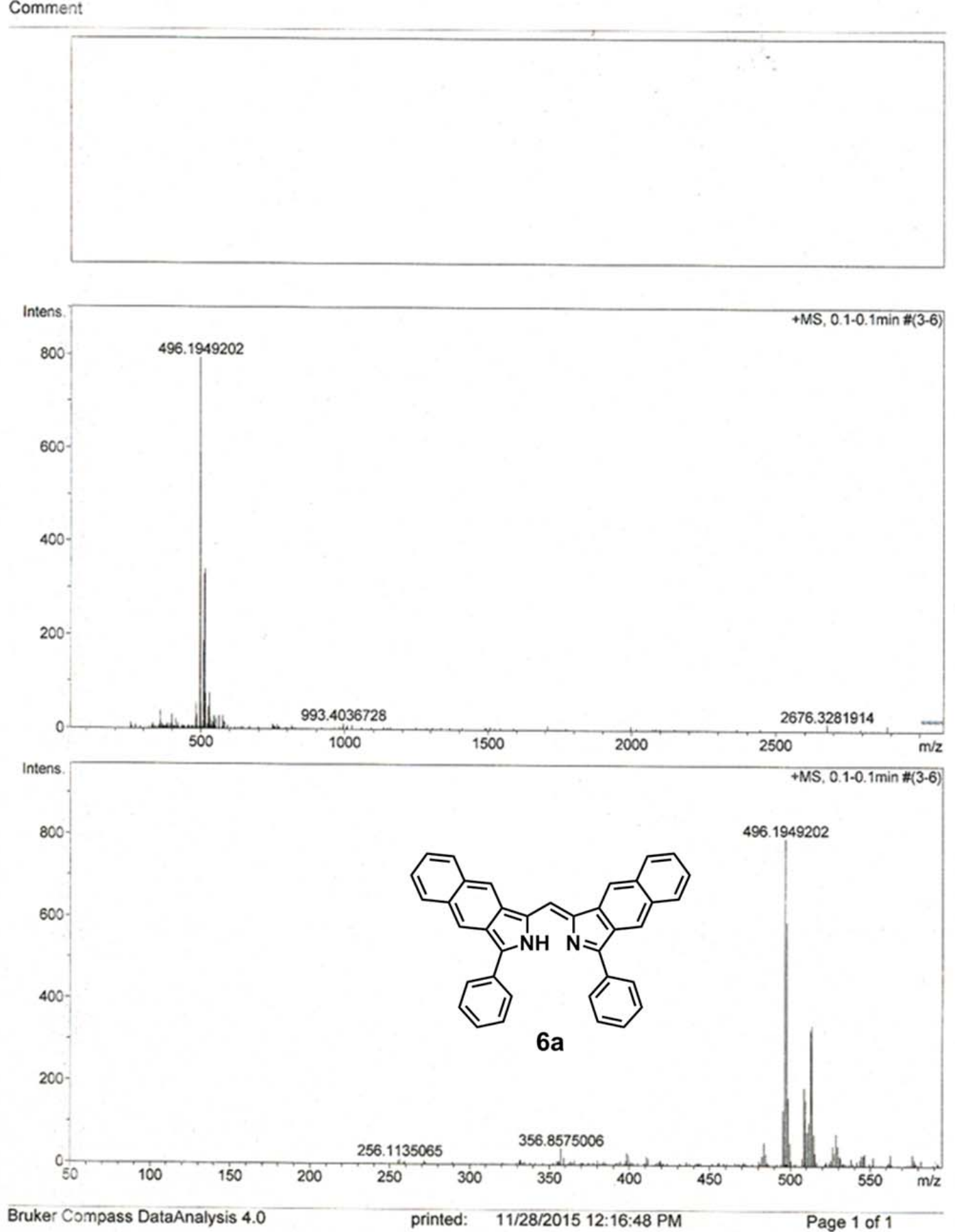

Figure S15. High-resolution APCI mass spectrum of $\mathbf{6 a}$. 


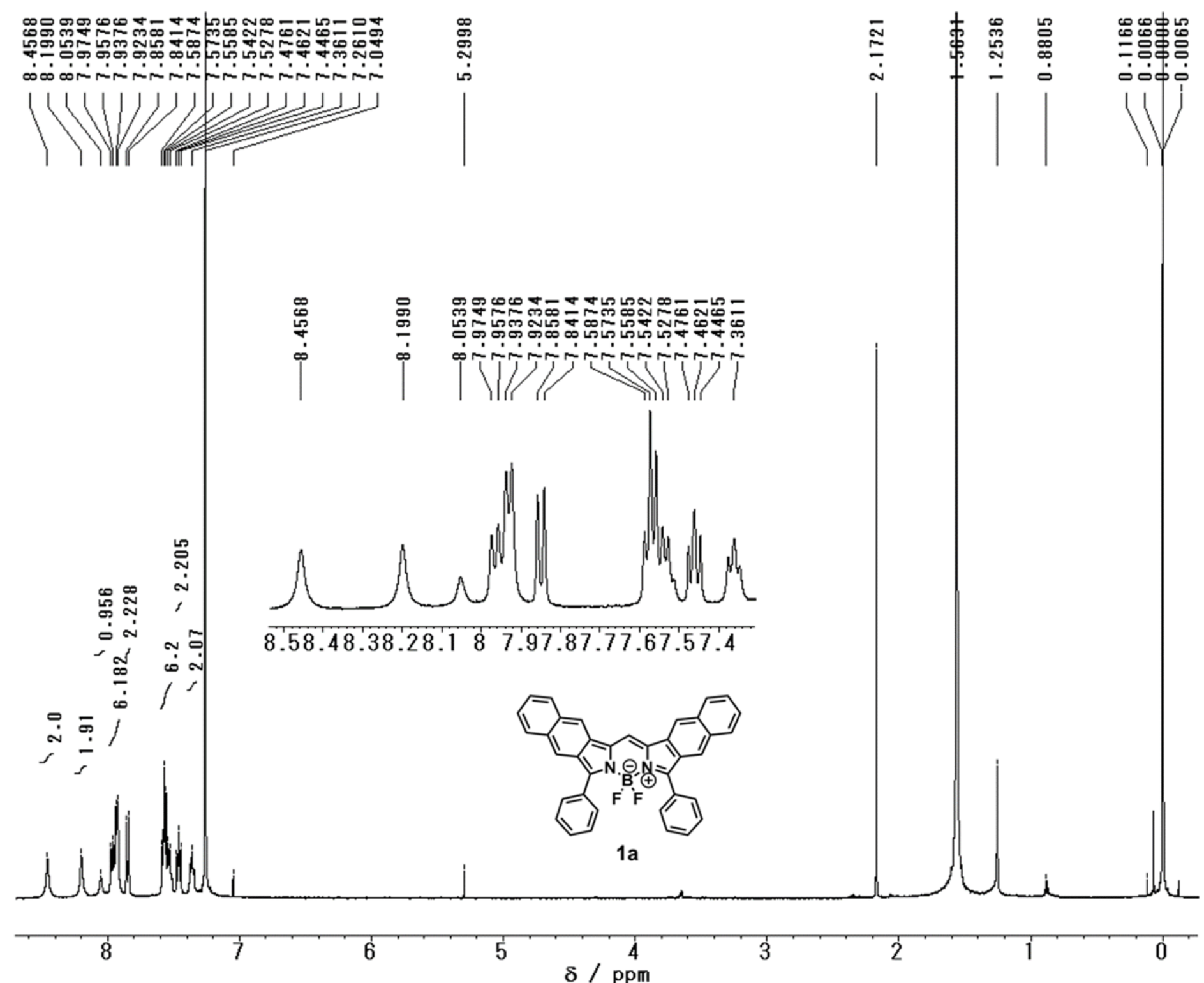

Figure S16. ${ }^{1} \mathrm{H}$ NMR spectrum of $1 \mathbf{a}(500 \mathrm{MHz})$ in $\mathrm{CDCl}_{3}$. 


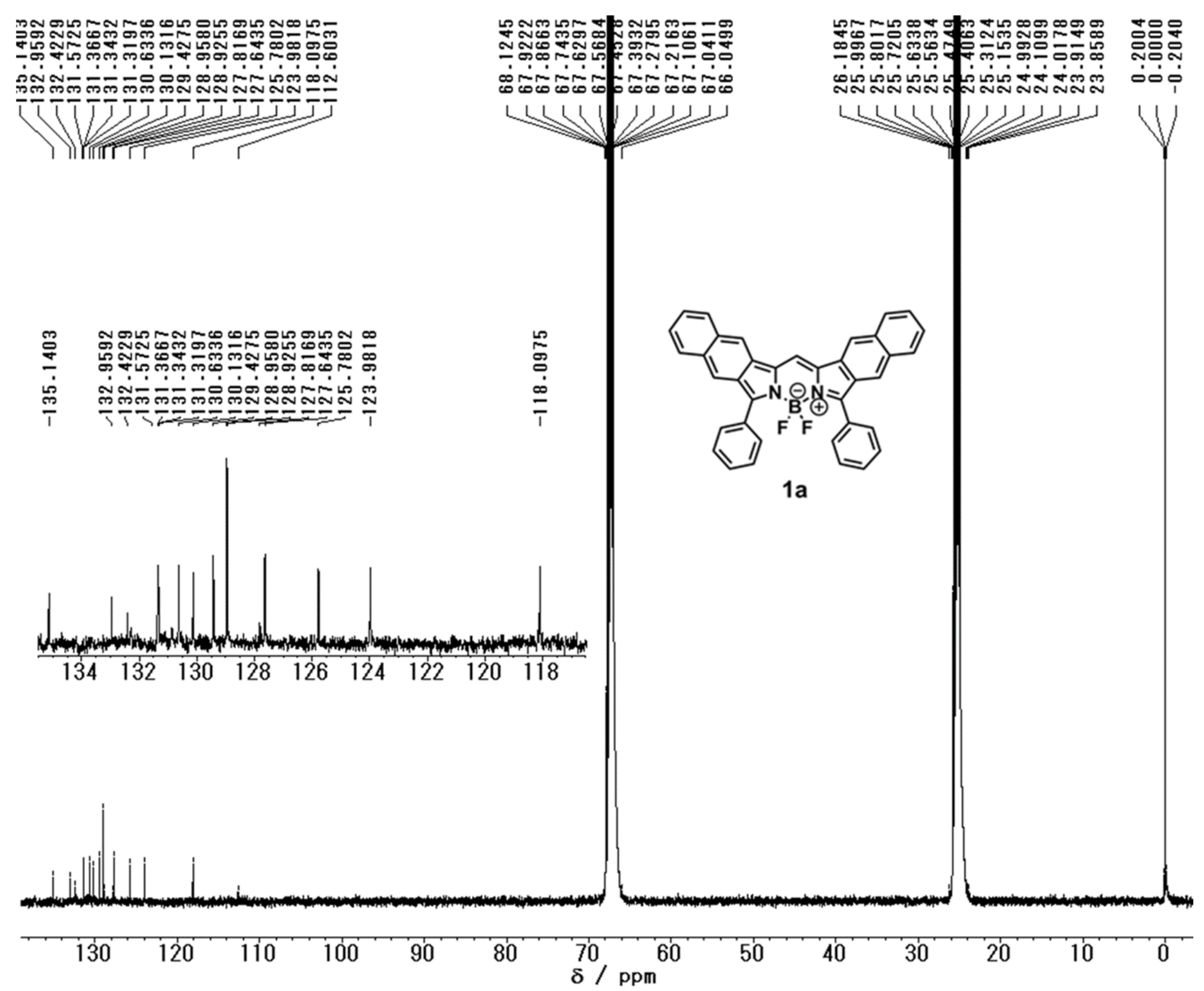

Figure S17. ${ }^{13} \mathrm{C}$ NMR spectrum of $1 \mathbf{a}(500 \mathrm{MHz})$ in THF- $d_{8}$. 
[ Mass Spectrum ]

Data : 20140726 EXP181-001 Date : 26-Jul-2014 11:01

Instrument : MStation

Sample : -

Inlet : Direct Ion Mode : FAB+

Spectrum Type : Normal Ion [MF-Linear]

Scan\# : $(3,8)$

BP : $\mathrm{m} / \mathrm{z} 154 \quad$ Int. : 18.47 (193716)

Output $\mathrm{m} / \mathrm{z}$ range : 50 to 578 Cut Level : $0.00 \%$

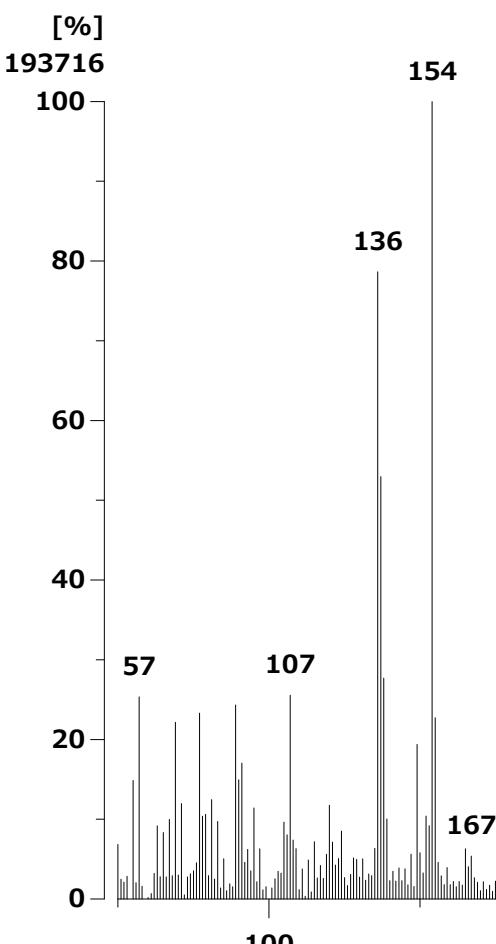

100

307

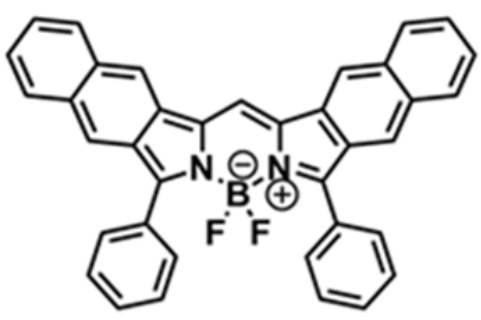

1a

Figure S18. FAB mass spectrum of 1 a. 


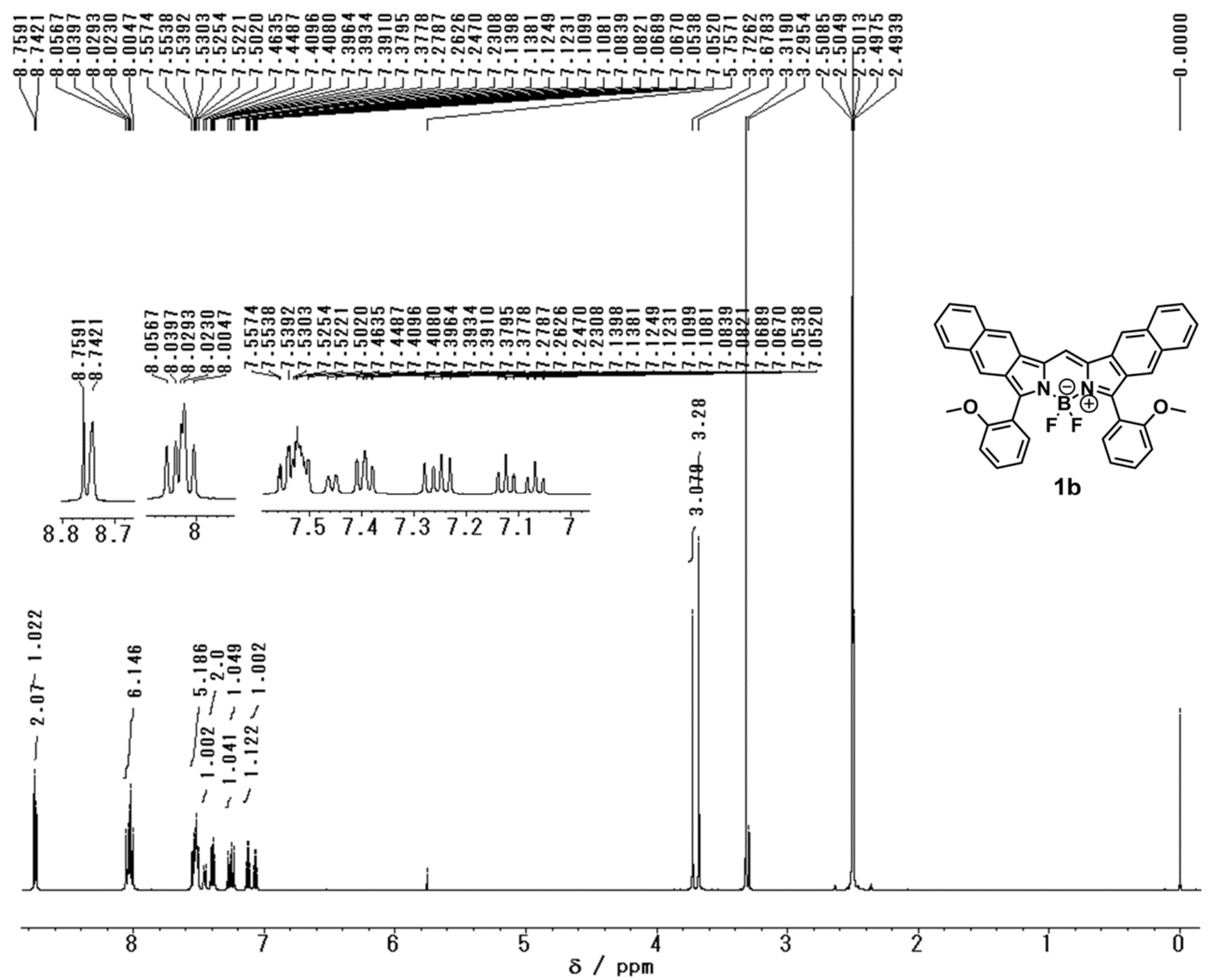

Figure S19. ${ }^{1} \mathrm{H}$ NMR spectrum of $\mathbf{1 b}(500 \mathrm{MHz})$ in DMSO- $d_{6}$. 


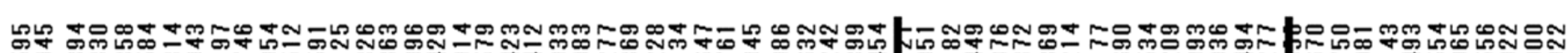

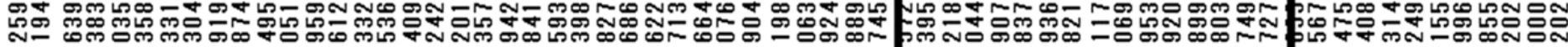

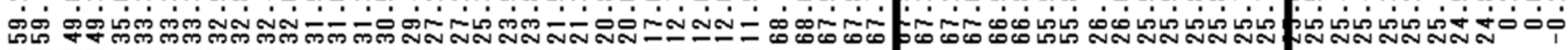

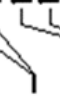

71 $111 \pi$

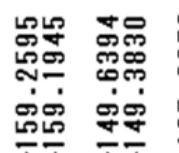

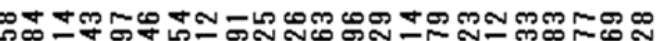

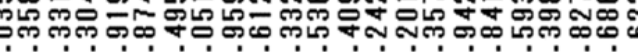

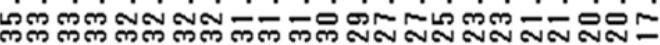

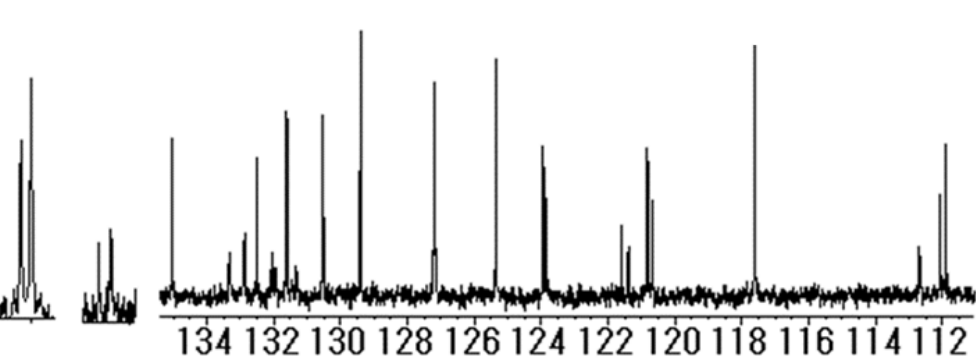

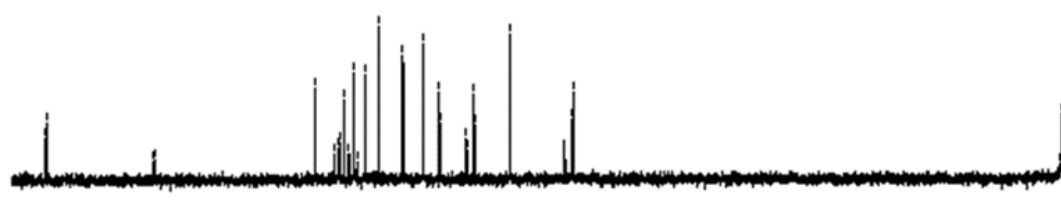

160

140

120

100

80

/ ppm

60

20

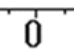

Figure S20. ${ }^{13} \mathrm{C}$ NMR spectrum of $\mathbf{1 b}(125 \mathrm{MHz})$ in THF- $d_{8}$. 
[ Mass Spectrum ]

Data : 20150831-EXP120-001 Date : 31-Aug-2015 21:19

Instrument : MStation

Sample : -

Inlet : Direct Ion Mode : FAB+

Spectrum Type : Normal Ion [MF-Linear]

Scan\# : $(4,9)$

BP : $m / z 154 \quad$ Int. : 14.21 (149009)

Output $\mathrm{m} / \mathrm{z}$ range : 50 to 653 Cut Level : $0.00 \%$

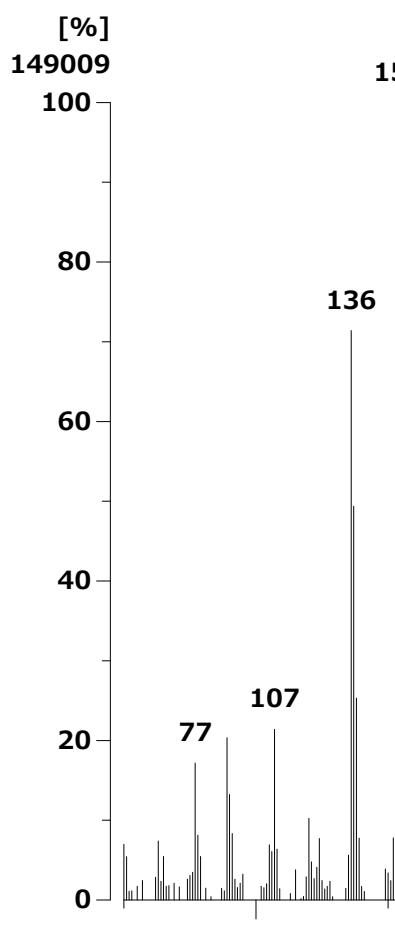

100

154

(1)

36

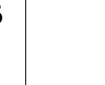

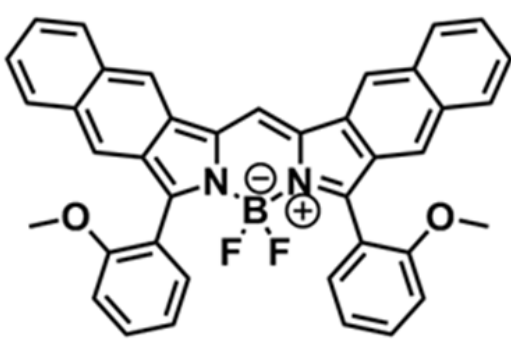

1b

Figure S21. FAB mass spectrum of $\mathbf{1 b}$. 


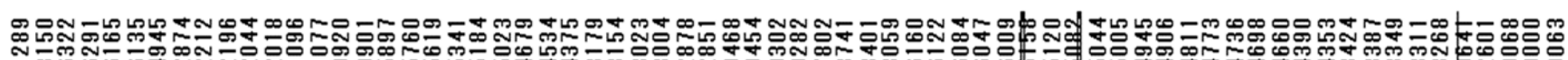

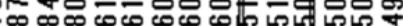

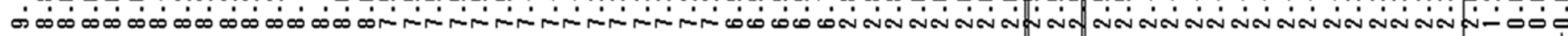

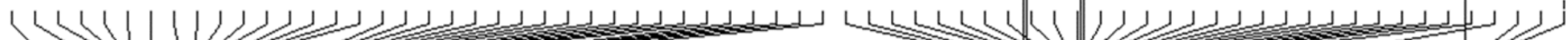

I 1 |

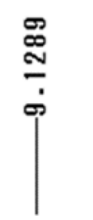

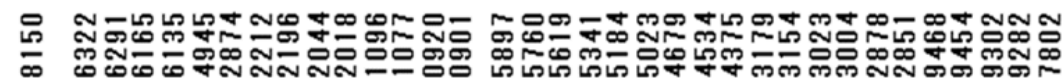

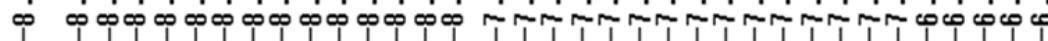
Y

$\mid$

9.29 .198 .98 .88 .78 .68 .58 .48 .38 .2
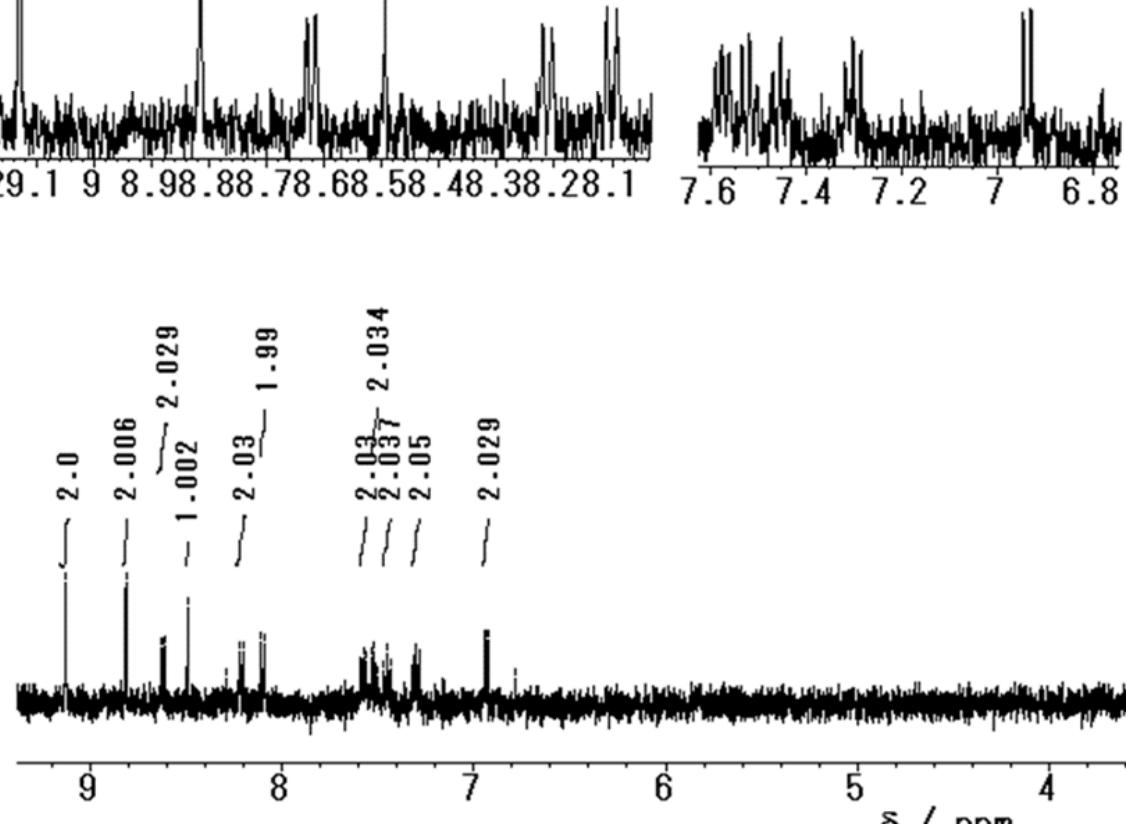

నุำ

in

111

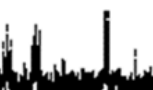

6

$\delta / \mathrm{ppm}$

Figure S22. ${ }^{1} \mathrm{H}$ NMR spectrum of $2(500 \mathrm{MHz})$ in DMSO- $d_{6}$.

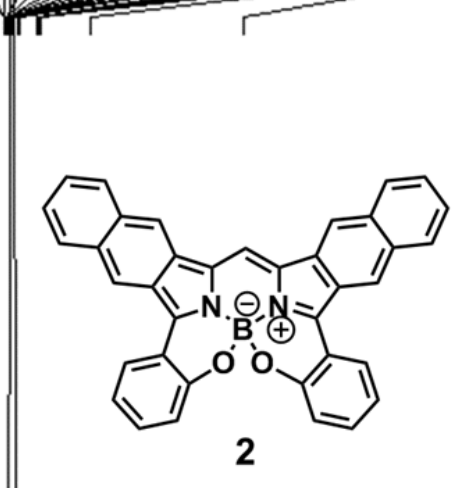


Generic Display Report
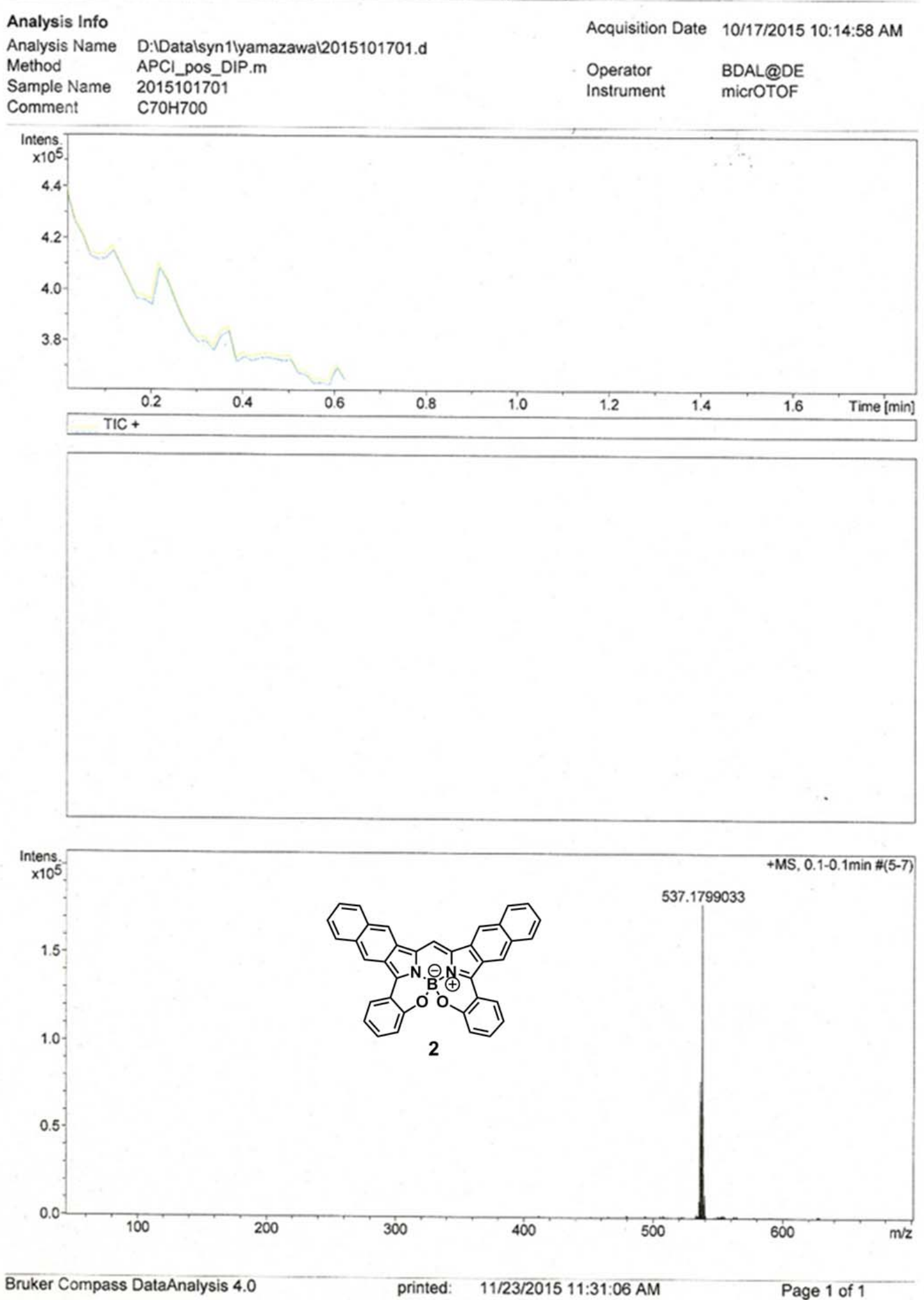

Figure S23. High-resolution APCI mass spectrum of 2. 


\section{Single crystal X-ray diffraction study}

\section{Data Collection}

A brown needle crystal of $\mathrm{C}_{37} \mathrm{H}_{23} \mathrm{BF}_{2} \mathrm{~N}_{2}$ having approximate dimensions of $0.570 \times 0.060 \times 0.040 \mathrm{~mm}$ was mounted on a glass fiber. All measurements were made on a Rigaku XtaLAB P200 diffractometer using multi-layer mirror monochromated Mo-K $\alpha$ radiation. The crystal-to-detector distance was $44.94 \mathrm{~mm}$. Cell constants and an orientation matrix for data collection corresponded to a primitive monoclinic cell with dimensions:

$$
\begin{aligned}
& a=12.119(4) \AA \\
& b=7.848(2) \AA \\
& c=27.185(8) \AA \\
& \beta=90.261(9)^{\circ} \\
& V=2585.5(13) \AA^{3}
\end{aligned}
$$

For $\mathrm{Z}=4$ and F.W. $=544.41$, the calculated density is $1.398 \mathrm{~g} / \mathrm{cm}^{3}$. The reflection conditions of:

h01: $\mathrm{h}+\mathrm{l}=2 \mathrm{n}$

0k0: $\mathrm{k}=2 \mathrm{n}$

uniquely determine the space group to be: $\mathrm{P} 2 / \mathrm{n}(\# 14)$

The data were collected at a temperature of $-160 \pm 1{ }^{\circ} \mathrm{C}$ to a maximum $2 \theta$ value of $55.1^{\circ}$. A total of 720 oscillation images were collected. A sweep of data was done using $\omega$ scans from -98.0 to $82.0^{\circ}$ in $0.25^{\circ}$ step, at $\chi=44.0^{\circ}$ and $\varphi=-180.0^{\circ}$. The exposure rate was 80.0 [sec. $/^{\circ}$ ]. The detector swing angle was $-8.01^{\circ}$. The crystal-to-detector distance was $44.94 \mathrm{~mm}$. Readout was performed in the $0.172 \mathrm{~mm}$ pixel mode.

\section{Data Reduction}

Of the 11077 reflections were collected, where 4995 were unique $\left(\mathrm{R}_{\text {int }}=0.0305\right)$; equivalent reflections were merged. Data were collected and processed using CrystalClear (Rigaku). ${ }^{1}$

The linear absorption coefficient, $\mu$, for Mo-K $\alpha$ radiation is $0.910 \mathrm{~cm}^{-1}$. An empirical absorption correction was applied which resulted in transmission factors ranging from 0.798 to 0.996 . The data were corrected for Lorentz and polarization effects.

\section{$\underline{\text { Structure Solution and Refinement }}$}

The structure was solved by direct methods ${ }^{2}$ and expanded using Fourier techniques. The non-hydrogen atoms were refined anisotropically. Hydrogen atoms were refined using the riding model. The final cycle of full-matrix least-squares refinement ${ }^{3}$ on $\mathrm{F}^{2}$ was based on 4995 observed reflections and 379 variable parameters and converged (largest parameter shift was 0.00 times its esd) with unweighted and weighted agreement factors of: 
$\mathrm{R} 1=\Sigma\left(\left|\mathrm{F}_{\mathrm{o}}\right|-\left|\mathrm{F}_{\mathrm{c}}\right| / / \Sigma\left|\mathrm{F}_{\mathrm{o}}\right|=0.0387\right.$

$\mathrm{wR} 2=\left[\Sigma\left(\mathrm{w}\left(\mathrm{F}_{\mathrm{o}}{ }^{2}-\mathrm{F}_{\mathrm{c}}\right)^{2}\right) / \Sigma \mathrm{w}\left(\mathrm{F}_{\mathrm{o}}{ }^{2}\right)^{2}\right]^{1 / 2}=0.1070$

The goodness of fit ${ }^{4}$ was 1.04. Unit weights were used. Plots of $\Sigma \mathrm{w}\left(\left|\mathrm{F}_{\mathrm{o}}\right|-\left|\mathrm{F}_{\mathrm{c}}\right|\right)^{2}$ versus $\left|\mathrm{F}_{\mathrm{o}}\right|$, reflection order in data collection, $\sin \theta / \lambda$ and various classes of indices showed no unusual trends. The maximum and minimum peaks on the final difference Fourier map corresponded to 0.26 and $-0.22 \mathrm{e}^{-} / \AA^{3}$, respectively.

Neutral atom scattering factors were taken from International Tables for Crystallography (IT), Vol. C, Table 6.1.1.4 . Anomalous dispersion effects were included in Fcalc $^{6}$; the values for $\Delta \mathrm{f}^{\prime}$ and $\Delta \mathrm{f}^{\prime \prime}$ were those of Creagh and McAuley ${ }^{7}$. The values for the mass attenuation coefficients are those of Creagh and Hubbell ${ }^{8}$. All calculations were performed using the CrystalStructure ${ }^{9}$ crystallographic software package except for refinement, which was performed using SHELXL2013 ${ }^{10}$.

\section{References}

(1) CrystalClear: Data Collection and Processing Software, Rigaku Corporation (1998-2014). Tokyo 196-8666, Japan.

(2) SHELXS2013: Sheldrick, G. M. Acta Cryst. 2008, A64, 112-122.

(3) Least Squares function minimized: (SHELXL2013)

$$
\Sigma w\left(\mathrm{~F}_{\mathrm{o}}{ }^{2}-\mathrm{F}_{\mathrm{c}}{ }^{2}\right)^{2} \quad \text { where } \mathrm{w}=\text { Least Squares weights. }
$$

(4) Goodness of fit is defined as:

$$
\left[\Sigma w\left(\mathrm{~F}_{\mathrm{o}}^{2}-\mathrm{F}_{\mathrm{c}}^{2}\right)^{2} /\left(\mathrm{N}_{\mathrm{o}}-\mathrm{N}_{\mathrm{v}}\right)\right]^{1 / 2}
$$

where: $\quad N_{o}=$ number of observations, $N_{v}=$ number of variables

(5) International Tables for Crystallography, Vol. C (1992). Ed. A. J. C. Wilson, Kluwer Academic Publishers, Dordrecht, Netherlands, Table 6.1.1.4, p. 572.

(6) Ibers, J. A.; Hamilton, W. C. Acta Crystallogr. 1964, 17, 781-782.

(7) Creagh, D. C.; McAuley, W. J. "International Tables for Crystallography", Vol C, (A. J. C. Wilson, ed.), Kluwer Academic Publishers, Boston, Table 4.2.6.8, 1992, 219-222.

(8) Creagh, D. C.; Hubbell, J.H. "International Tables for Crystallography", Vol C, (A.J.C. Wilson, ed.), Kluwer Academic Publishers, Boston, Table 4.2.4.3, 1992, 200-206.

(9) CrystalStructure 4.1: Crystal Structure Analysis Package, Rigaku Corporation (2000-2014). Tokyo 196-8666, Japan.

(10) SHELXL2013: Sheldrick, G. M. Acta Cryst. 2008, A64, 112-122. 


\section{A. Crystal Data}

Empirical Formula

Formula Weight

Crystal Color, Habit

Crystal Dimensions

Crystal System

Lattice Type

Lattice Parameters

Space Group

$\mathrm{Z}$ value

$\mathrm{D}_{\text {calc }}$

$\mathrm{F}_{000}$

$\mu(\operatorname{MoK} \alpha)$
$\mathrm{C}_{37} \mathrm{H}_{23} \mathrm{BF}_{2} \mathrm{~N}_{2}$

544.41

brown, needle

$0.570 \times 0.060 \times 0.040 \mathrm{~mm}$

monoclinic

Primitive

$$
\begin{aligned}
& \mathrm{a}=12.119(4) \AA \\
& \mathrm{b}=7.848(2) \AA \\
& \mathrm{c}=27.185(8) \AA \\
& \beta=90.261(9){ }^{\circ} \\
& \mathrm{V}=2585.5(13) \AA^{3}
\end{aligned}
$$

$\mathrm{P} 2{ }_{1} / \mathrm{n}(\# 14)$

4

$1.398 \mathrm{~g} / \mathrm{cm}^{3}$

1128.00

$0.910 \mathrm{~cm}^{-1}$ 
B. Intensity Measurements

Diffractometer

Radiation

Voltage, Current

Temperature

Detector Aperture

Data Images

$\omega$ oscillation Range $(\chi=44.0, \phi=-180.0)$

Exposure Rate

Detector Swing Angle

Detector Position

Pixel Size

$2 \theta_{\max }$

No. of Reflections Measured

Corrections
XtaLAB P200

$\operatorname{MoK} \alpha(\lambda=0.71075 \AA)$

multi-layer mirror monochromated

$50 \mathrm{kV}, 24 \mathrm{~mA}$

$-160.0^{\circ} \mathrm{C}$

$83.8 \times 70.0 \mathrm{~mm}$

720 exposures

$-98.0-82.0^{\circ}$

$80.0 \mathrm{sec} . /^{\circ}$

$-8.01^{\circ}$

$44.94 \mathrm{~mm}$

$0.172 \mathrm{~mm}$

$55.1^{\circ}$

Total: 11077

Unique: $4995\left(\mathrm{R}_{\mathrm{int}}=0.0305\right)$

Lorentz-polarization

Absorption

(trans. factors: $0.798-0.996$ ) 
Structure Solution

Refinement

Function Minimized

Least Squares Weights

$2 \theta_{\max }$ cutoff

Anomalous Dispersion

No. Observations (All reflections)

No. Variables

Reflection/Parameter Ratio

Residuals: R1 (I>2.00б(I))

Residuals: R (All reflections)

Residuals: wR2 (All reflections)

Goodness of Fit Indicator

Max Shift/Error in Final Cycle

Maximum peak in Final Diff. Map

Minimum peak in Final Diff. Map
Direct Methods (SHELXS2013)

Full-matrix least-squares on $\mathrm{F}^{2}$

$\Sigma \mathrm{W}\left(\mathrm{F}_{\mathrm{o}}{ }^{2}-\mathrm{F}_{\mathrm{c}}{ }^{2}\right)^{2}$

$\mathrm{w}=1 /\left[\sigma^{2}\left(\mathrm{~F}_{\mathrm{o}}{ }^{2}\right)+(0.0593 \cdot \mathrm{P})^{2}\right.$

$+0.5767 \cdot \mathrm{P}]$

where $\mathrm{P}=\left(\operatorname{Max}\left(\mathrm{F}_{\mathrm{o}}{ }^{2}, 0\right)+2 \mathrm{~F}_{\mathrm{c}}{ }^{2}\right) / 3$

$55.1^{\circ}$

All non-hydrogen atoms

4995

379

13.18

0.0387

0.0504

0.1070

1.041

0.001

$0.26 \mathrm{e}^{-} / \AA^{3}$

$-0.22 \mathrm{e}^{-} / \AA^{3}$ 
Figure S24. (a) Atom numbering Scheme of 1a and (b) its bond lengths and bond angles.

(a)

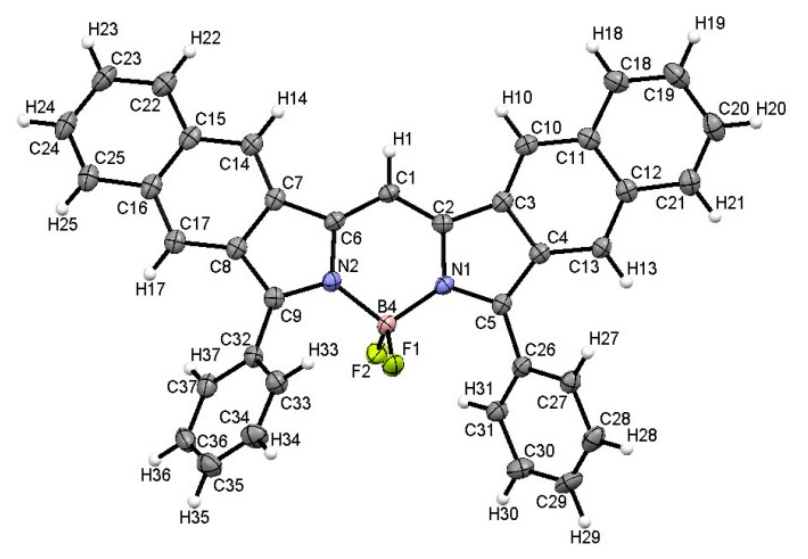

(b)

Bond lengths $(\AA)$

\begin{tabular}{|c|c|c|c|c|c|}
\hline atom & atom & distance & atom & atom & distance \\
\hline F1 & B4 & $1.3695(19)$ & $\mathrm{F} 2$ & B4 & $1.3872(19)$ \\
\hline $\mathrm{N} 1$ & $\mathrm{C} 2$ & $1.3892(18)$ & $\mathrm{N} 1$ & $\mathrm{C} 5$ & $1.3607(18)$ \\
\hline N1 & B4 & $1.573(2)$ & $\mathrm{N} 2$ & C6 & $1.3973(18)$ \\
\hline $\mathrm{N} 2$ & C9 & $1.3491(18)$ & $\mathrm{N} 2$ & B4 & $1.588(2)$ \\
\hline $\mathrm{C} 1$ & $\mathrm{C} 2$ & $1.3901(19)$ & $\mathrm{C} 1$ & C6 & $1.3768(19)$ \\
\hline $\mathrm{C} 2$ & $\mathrm{C} 3$ & $1.4262(19)$ & $\mathrm{C} 3$ & $\mathrm{C} 4$ & $1.4325(19)$ \\
\hline $\mathrm{C} 3$ & $\mathrm{C} 10$ & $1.382(2)$ & $\mathrm{C} 4$ & $\mathrm{C} 5$ & $1.4259(19)$ \\
\hline $\mathrm{C} 4$ & $\mathrm{C} 13$ & $1.3933(19)$ & $\mathrm{C} 5$ & $\mathrm{C} 26$ & $1.4704(19)$ \\
\hline C6 & $\mathrm{C} 7$ & $1.434(2)$ & $\mathrm{C} 7$ & $\mathrm{C} 8$ & $1.426(2)$ \\
\hline $\mathrm{C} 7$ & $\mathrm{C} 14$ & $1.380(2)$ & $\mathrm{C} 8$ & C9 & $1.437(2)$ \\
\hline $\mathrm{C} 8$ & $\mathrm{C} 17$ & $1.392(2)$ & $\mathrm{C} 9$ & $\mathrm{C} 32$ & $1.465(2)$ \\
\hline $\mathrm{C} 10$ & $\mathrm{C} 11$ & $1.404(2)$ & $\mathrm{C} 11$ & $\mathrm{C} 12$ & $1.442(2)$ \\
\hline $\mathrm{C} 11$ & $\mathrm{C} 18$ & $1.422(2)$ & $\mathrm{C} 12$ & $\mathrm{C} 13$ & $1.395(2)$ \\
\hline $\mathrm{C} 12$ & $\mathrm{C} 21$ & $1.429(2)$ & C14 & $\mathrm{C} 15$ & $1.406(2)$ \\
\hline $\mathrm{C} 15$ & $\mathrm{C} 16$ & $1.433(2)$ & $\mathrm{C} 15$ & $\mathrm{C} 22$ & $1.425(2)$ \\
\hline $\mathrm{C} 16$ & $\mathrm{C} 17$ & $1.404(2)$ & C16 & $\mathrm{C} 25$ & $1.432(2)$ \\
\hline $\mathrm{C} 18$ & C19 & $1.361(2)$ & C19 & $\mathrm{C} 20$ & $1.418(2)$ \\
\hline $\mathrm{C} 20$ & $\mathrm{C} 21$ & $1.357(2)$ & $\mathrm{C} 22$ & $\mathrm{C} 23$ & $1.368(2)$ \\
\hline $\mathrm{C} 23$ & $\mathrm{C} 24$ & $1.411(2)$ & $\mathrm{C} 24$ & $\mathrm{C} 25$ & $1.363(2)$ \\
\hline $\mathrm{C} 26$ & $\mathrm{C} 27$ & $1.403(2)$ & $\mathrm{C} 26$ & $\mathrm{C} 31$ & $1.401(2)$ \\
\hline $\mathrm{C} 27$ & $\mathrm{C} 28$ & $1.384(2)$ & $\mathrm{C} 28$ & $\mathrm{C} 29$ & $1.379(2)$ \\
\hline $\mathrm{C} 29$ & $\mathrm{C} 30$ & $1.387(2)$ & $\mathrm{C} 30$ & $\mathrm{C} 31$ & $1.385(2)$ \\
\hline $\mathrm{C} 32$ & $\mathrm{C} 33$ & $1.402(2)$ & $\mathrm{C} 32$ & $\mathrm{C} 37$ & $1.401(2)$ \\
\hline $\mathrm{C} 33$ & $\mathrm{C} 34$ & $1.380(2)$ & $\mathrm{C} 34$ & $\mathrm{C} 35$ & $1.380(2)$ \\
\hline $\mathrm{C} 35$ & $\mathrm{C} 36$ & $1.386(2)$ & $\mathrm{C} 36$ & $\mathrm{C} 37$ & $1.385(2)$ \\
\hline
\end{tabular}


Bond lengths involving hydrogens $(\AA)$

\begin{tabular}{|c|c|c|c|c|c|}
\hline atom & atom & distance & atom & atom & distance \\
\hline $\mathrm{C} 1$ & H1 & 0.950 & $\mathrm{C} 10$ & $\mathrm{H} 10$ & 0.950 \\
\hline $\mathrm{C} 13$ & $\mathrm{H} 13$ & 0.950 & $\mathrm{C} 14$ & H14 & 0.950 \\
\hline $\mathrm{C} 17$ & $\mathrm{H} 17$ & 0.950 & $\mathrm{C} 18$ & H1 8 & 0.950 \\
\hline $\mathrm{C} 19$ & H19 & 0.950 & $\mathrm{C} 20$ & $\mathrm{H} 20$ & 0.950 \\
\hline $\mathrm{C} 21$ & $\mathrm{H} 21$ & 0.950 & C22 & $\mathrm{H} 22$ & 0.950 \\
\hline $\mathrm{C} 23$ & $\mathrm{H} 23$ & 0.950 & $\mathrm{C} 24$ & $\mathrm{H} 24$ & 0.950 \\
\hline $\mathrm{C} 25$ & $\mathrm{H} 25$ & 0.950 & $\mathrm{C} 27$ & $\mathrm{H} 27$ & 0.950 \\
\hline $\mathrm{C} 28$ & $\mathrm{H} 28$ & 0.950 & $\mathrm{C} 29$ & $\mathrm{H} 29$ & 0.950 \\
\hline $\mathrm{C} 30$ & $\mathrm{H} 30$ & 0.950 & $\mathrm{C} 31$ & H31 & 0.950 \\
\hline $\mathrm{C} 33$ & $\mathrm{H} 33$ & 0.950 & $\mathrm{C} 34$ & H34 & 0.950 \\
\hline $\mathrm{C} 35$ & H35 & 0.950 & $\mathrm{C} 36$ & H36 & 0.950 \\
\hline C37 & $\mathrm{H} 37$ & 0.950 & & & \\
\hline
\end{tabular}


Bond angles $\left(^{\circ}\right)$

\begin{tabular}{|c|c|c|c|c|c|c|c|}
\hline atom & atom & atom & angle & atom & atom & atom & angle \\
\hline $\mathrm{C} 2$ & N1 & C5 & $109.98(11)$ & $\mathrm{C} 2$ & N1 & B4 & $122.87(11)$ \\
\hline $\mathrm{C} 5$ & $\mathrm{~N} 1$ & B4 & $127.12(11)$ & C6 & $\mathrm{N} 2$ & C9 & $110.01(11)$ \\
\hline C6 & $\mathrm{N} 2$ & B4 & $120.95(11)$ & C9 & N2 & B4 & $126.04(11)$ \\
\hline $\mathrm{C} 2$ & $\mathrm{C} 1$ & C6 & $122.67(13)$ & $\mathrm{N} 1$ & $\mathrm{C} 2$ & $\mathrm{C} 1$ & $120.93(12)$ \\
\hline N1 & $\mathrm{C} 2$ & $\mathrm{C} 3$ & $108.20(11)$ & $\mathrm{C} 1$ & $\mathrm{C} 2$ & $\mathrm{C} 3$ & $130.87(13)$ \\
\hline $\mathrm{C} 2$ & $\mathrm{C} 3$ & $\mathrm{C} 4$ & $106.20(12)$ & $\mathrm{C} 2$ & $\mathrm{C} 3$ & $\mathrm{C} 10$ & $132.91(13)$ \\
\hline $\mathrm{C} 4$ & $\mathrm{C} 3$ & $\mathrm{C} 10$ & $120.86(13)$ & $\mathrm{C} 3$ & $\mathrm{C} 4$ & $\mathrm{C} 5$ & $107.21(12)$ \\
\hline $\mathrm{C} 3$ & $\mathrm{C} 4$ & $\mathrm{C} 13$ & $120.45(12)$ & $\mathrm{C} 5$ & $\mathrm{C} 4$ & $\mathrm{C} 13$ & $132.19(13)$ \\
\hline N1 & $\mathrm{C} 5$ & $\mathrm{C} 4$ & $108.40(12)$ & $\mathrm{N} 1$ & $\mathrm{C} 5$ & $\mathrm{C} 26$ & $126.17(12)$ \\
\hline $\mathrm{C} 4$ & $\mathrm{C} 5$ & $\mathrm{C} 26$ & $125.32(12)$ & N2 & C6 & $\mathrm{C} 1$ & $120.94(12)$ \\
\hline N2 & C6 & $\mathrm{C} 7$ & $107.77(12)$ & $\mathrm{C} 1$ & C6 & $\mathrm{C} 7$ & $131.29(13)$ \\
\hline C6 & $\mathrm{C} 7$ & $\mathrm{C} 8$ & $106.52(12)$ & C6 & $\mathrm{C} 7$ & $\mathrm{C} 14$ & $132.84(13)$ \\
\hline $\mathrm{C} 8$ & $\mathrm{C} 7$ & $\mathrm{C} 14$ & $120.61(13)$ & $\mathrm{C} 7$ & $\mathrm{C} 8$ & $\mathrm{C} 9$ & $106.85(12)$ \\
\hline $\mathrm{C} 7$ & $\mathrm{C} 8$ & $\mathrm{C} 17$ & $120.99(13)$ & $\mathrm{C} 9$ & $\mathrm{C} 8$ & $\mathrm{C} 17$ & $132.15(13)$ \\
\hline $\mathrm{N} 2$ & C9 & $\mathrm{C} 8$ & $108.81(12)$ & $\mathrm{N} 2$ & $\mathrm{C} 9$ & $\mathrm{C} 32$ & $125.46(13)$ \\
\hline $\mathrm{C} 8$ & C9 & $\mathrm{C} 32$ & $125.63(12)$ & $\mathrm{C} 3$ & $\mathrm{C} 10$ & $\mathrm{C} 11$ & $118.92(13)$ \\
\hline $\mathrm{C} 10$ & C11 & $\mathrm{C} 12$ & $120.35(13)$ & $\mathrm{C} 10$ & $\mathrm{C} 11$ & $\mathrm{C} 18$ & $121.74(13)$ \\
\hline $\mathrm{C} 12$ & $\mathrm{C} 11$ & $\mathrm{C} 18$ & $117.91(13)$ & $\mathrm{C} 11$ & $\mathrm{C} 12$ & $\mathrm{C} 13$ & $120.00(13)$ \\
\hline $\mathrm{C} 11$ & $\mathrm{C} 12$ & $\mathrm{C} 21$ & $118.51(13)$ & $\mathrm{C} 13$ & $\mathrm{C} 12$ & $\mathrm{C} 21$ & $121.49(13)$ \\
\hline $\mathrm{C} 4$ & $\mathrm{C} 13$ & $\mathrm{C} 12$ & $119.21(13)$ & $\mathrm{C} 7$ & $\mathrm{C} 14$ & $\mathrm{C} 15$ & $119.27(13)$ \\
\hline $\mathrm{C} 14$ & $\mathrm{C} 15$ & $\mathrm{C} 16$ & $120.11(13)$ & $\mathrm{C} 14$ & $\mathrm{C} 15$ & $\mathrm{C} 22$ & $121.16(13)$ \\
\hline $\mathrm{C} 16$ & $\mathrm{C} 15$ & $\mathrm{C} 22$ & $118.66(13)$ & $\mathrm{C} 15$ & $\mathrm{C} 16$ & $\mathrm{C} 17$ & $120.24(13)$ \\
\hline $\mathrm{C} 15$ & $\mathrm{C} 16$ & $\mathrm{C} 25$ & $118.46(14)$ & $\mathrm{C} 17$ & $\mathrm{C} 16$ & $\mathrm{C} 25$ & $121.28(14)$ \\
\hline $\mathrm{C} 8$ & $\mathrm{C} 17$ & $\mathrm{C} 16$ & $118.66(13)$ & $\mathrm{C} 11$ & $\mathrm{C} 18$ & $\mathrm{C} 19$ & $121.48(14)$ \\
\hline $\mathrm{C} 18$ & $\mathrm{C} 19$ & $\mathrm{C} 20$ & $120.58(15)$ & C19 & $\mathrm{C} 20$ & $\mathrm{C} 21$ & $120.07(15)$ \\
\hline $\mathrm{C} 12$ & $\mathrm{C} 21$ & $\mathrm{C} 20$ & $121.36(14)$ & $\mathrm{C} 15$ & $\mathrm{C} 22$ & $\mathrm{C} 23$ & $120.90(14)$ \\
\hline $\mathrm{C} 22$ & $\mathrm{C} 23$ & $\mathrm{C} 24$ & $120.45(15)$ & $\mathrm{C} 23$ & $\mathrm{C} 24$ & $\mathrm{C} 25$ & $120.63(15)$ \\
\hline $\mathrm{C} 16$ & $\mathrm{C} 25$ & $\mathrm{C} 24$ & $120.81(15)$ & $\mathrm{C} 5$ & $\mathrm{C} 26$ & $\mathrm{C} 27$ & $118.88(12)$ \\
\hline $\mathrm{C} 5$ & $\mathrm{C} 26$ & $\mathrm{C} 31$ & $122.43(12)$ & $\mathrm{C} 27$ & $\mathrm{C} 26$ & $\mathrm{C} 31$ & $118.53(13)$ \\
\hline $\mathrm{C} 26$ & $\mathrm{C} 27$ & $\mathrm{C} 28$ & $120.60(13)$ & $\mathrm{C} 27$ & $\mathrm{C} 28$ & $\mathrm{C} 29$ & $120.26(15)$ \\
\hline $\mathrm{C} 28$ & $\mathrm{C} 29$ & $\mathrm{C} 30$ & $119.92(15)$ & $\mathrm{C} 29$ & $\mathrm{C} 30$ & $\mathrm{C} 31$ & $120.46(14)$ \\
\hline $\mathrm{C} 26$ & $\mathrm{C} 31$ & $\mathrm{C} 30$ & $120.23(13)$ & $\mathrm{C} 9$ & $\mathrm{C} 32$ & $\mathrm{C} 33$ & $121.65(12)$ \\
\hline $\mathrm{C} 9$ & $\mathrm{C} 32$ & $\mathrm{C} 37$ & $119.86(13)$ & $\mathrm{C} 33$ & $\mathrm{C} 32$ & $\mathrm{C} 37$ & $118.36(13)$ \\
\hline $\mathrm{C} 32$ & $\mathrm{C} 33$ & $\mathrm{C} 34$ & $120.77(13)$ & $\mathrm{C} 33$ & $\mathrm{C} 34$ & $\mathrm{C} 35$ & $120.37(15)$ \\
\hline $\mathrm{C} 34$ & $\mathrm{C} 35$ & $\mathrm{C} 36$ & $119.70(16)$ & $\mathrm{C} 35$ & $\mathrm{C} 36$ & $\mathrm{C} 37$ & $120.55(15)$ \\
\hline $\mathrm{C} 32$ & $\mathrm{C} 37$ & $\mathrm{C} 36$ & $120.24(14)$ & $\mathrm{F} 1$ & B4 & $\mathrm{F} 2$ & $111.64(12)$ \\
\hline $\mathrm{F} 1$ & B4 & N1 & $108.79(12)$ & F1 & B4 & $\mathrm{N} 2$ & $112.63(13)$ \\
\hline F2 & B4 & N1 & $109.85(12)$ & F2 & B4 & $\mathrm{N} 2$ & $105.95(12)$ \\
\hline N1 & B4 & N2 & $107.87(11)$ & & & & \\
\hline
\end{tabular}


Bond angles involving hydrogens $\left(^{\circ}\right)$

\begin{tabular}{|c|c|c|c|c|c|c|c|}
\hline atom & atom & atom & angle & atom & atom & atom & angle \\
\hline $\mathrm{C} 2$ & $\mathrm{C} 1$ & H1 & 118.7 & C6 & $\mathrm{C} 1$ & H1 & 118.7 \\
\hline C3 & $\mathrm{C} 10$ & H10 & 120.5 & $\mathrm{C} 11$ & $\mathrm{C} 10$ & $\mathrm{H} 10$ & 120.5 \\
\hline $\mathrm{C} 4$ & $\mathrm{C} 13$ & $\mathrm{H} 13$ & 120.4 & $\mathrm{C} 12$ & $\mathrm{C} 13$ & $\mathrm{H} 13$ & 120.4 \\
\hline $\mathrm{C} 7$ & C14 & H14 & 120.4 & $\mathrm{C} 15$ & C14 & H14 & 120.4 \\
\hline C8 & $\mathrm{C} 17$ & H17 & 120.7 & $\mathrm{C} 16$ & $\mathrm{C} 17$ & H17 & 120.7 \\
\hline $\mathrm{C} 11$ & $\mathrm{C} 18$ & H18 & 119.3 & C19 & $\mathrm{C} 18$ & H18 & 119.3 \\
\hline $\mathrm{C} 18$ & C19 & H19 & 119.7 & C20 & $\mathrm{C} 19$ & H19 & 119.7 \\
\hline $\mathrm{C} 19$ & $\mathrm{C} 20$ & $\mathrm{H} 20$ & 120.0 & $\mathrm{C} 21$ & $\mathrm{C} 20$ & $\mathrm{H} 20$ & 120.0 \\
\hline $\mathrm{C} 12$ & $\mathrm{C} 21$ & $\mathrm{H} 21$ & 119.3 & $\mathrm{C} 20$ & $\mathrm{C} 21$ & $\mathrm{H} 21$ & 119.3 \\
\hline $\mathrm{C} 15$ & $\mathrm{C} 22$ & $\mathrm{H} 22$ & 119.6 & $\mathrm{C} 23$ & $\mathrm{C} 22$ & $\mathrm{H} 22$ & 119.5 \\
\hline $\mathrm{C} 22$ & $\mathrm{C} 23$ & $\mathrm{H} 23$ & 119.8 & $\mathrm{C} 24$ & $\mathrm{C} 23$ & $\mathrm{H} 23$ & 119.8 \\
\hline $\mathrm{C} 23$ & $\mathrm{C} 24$ & $\mathrm{H} 24$ & 119.7 & $\mathrm{C} 25$ & $\mathrm{C} 24$ & $\mathrm{H} 24$ & 119.7 \\
\hline $\mathrm{C} 16$ & $\mathrm{C} 25$ & $\mathrm{H} 25$ & 119.6 & $\mathrm{C} 24$ & $\mathrm{C} 25$ & $\mathrm{H} 25$ & 119.6 \\
\hline $\mathrm{C} 26$ & C27 & H27 & 119.7 & C28 & C27 & $\mathrm{H} 27$ & 119.7 \\
\hline C27 & $\mathrm{C} 28$ & $\mathrm{H} 28$ & 119.9 & C29 & C28 & $\mathrm{H} 28$ & 119.9 \\
\hline C28 & C29 & H29 & 120.1 & C30 & C29 & H29 & 120.0 \\
\hline C29 & $\mathrm{C} 30$ & $\mathrm{H} 30$ & 119.8 & C31 & $\mathrm{C} 30$ & $\mathrm{H} 30$ & 119.8 \\
\hline $\mathrm{C} 26$ & C31 & $\mathrm{H} 31$ & 119.9 & C30 & $\mathrm{C} 31$ & $\mathrm{H} 31$ & 119.9 \\
\hline $\mathrm{C} 32$ & C33 & $\mathrm{H} 33$ & 119.6 & C34 & C33 & $\mathrm{H} 33$ & 119.6 \\
\hline C33 & C34 & H34 & 119.8 & C35 & C34 & H34 & 119.8 \\
\hline C34 & C35 & H35 & 120.2 & C36 & C35 & H35 & 120.1 \\
\hline C35 & C36 & H36 & 119.7 & C37 & C36 & H36 & 119.7 \\
\hline $\mathrm{C} 32$ & C37 & H37 & 119.9 & C36 & C37 & H37 & 119.9 \\
\hline
\end{tabular}


Torsion Angles $\left({ }^{\circ}\right)$

(Those having bond angles $>160$ or $<20$ degrees are excluded.)

\begin{tabular}{|c|c|c|c|c|c|c|c|c|c|}
\hline atom1 & atom2 & atom3 & atom4 & angle & atom1 & atom2 & atom3 & atom4 & angle \\
\hline $\mathrm{C} 2$ & N1 & $\mathrm{C} 5$ & $\mathrm{C} 4$ & $1.02(14)$ & $\mathrm{C} 2$ & N1 & $\mathrm{C} 5$ & $\mathrm{C} 26$ & $-175.47(11)$ \\
\hline $\mathrm{C} 5$ & N1 & $\mathrm{C} 2$ & $\mathrm{C} 1$ & 179.92(11) & C5 & N1 & $\mathrm{C} 2$ & $\mathrm{C} 3$ & $-0.75(14)$ \\
\hline $\mathrm{C} 2$ & N1 & B4 & $\mathrm{F} 1$ & $-133.80(12)$ & $\mathrm{C} 2$ & N1 & B4 & $\mathrm{F} 2$ & $103.71(14)$ \\
\hline $\mathrm{C} 2$ & N1 & B4 & $\mathrm{N} 2$ & $-11.34(17)$ & B4 & N1 & $\mathrm{C} 2$ & $\mathrm{C} 1$ & $-1.85(18)$ \\
\hline B4 & N1 & $\mathrm{C} 2$ & $\mathrm{C} 3$ & $177.47(10)$ & $\mathrm{C} 5$ & N1 & B4 & $\mathrm{F} 1$ & $44.10(18)$ \\
\hline $\mathrm{C} 5$ & N1 & B4 & $\mathrm{F} 2$ & $-78.38(16)$ & $\mathrm{C} 5$ & N1 & B4 & $\mathrm{N} 2$ & $166.56(11)$ \\
\hline B4 & N1 & $\mathrm{C} 5$ & $\mathrm{C} 4$ & $-177.10(11)$ & B4 & N1 & $\mathrm{C} 5$ & $\mathrm{C} 26$ & $6.4(2)$ \\
\hline C6 & $\mathrm{N} 2$ & C9 & $\mathrm{C} 8$ & $2.13(14)$ & C6 & $\mathrm{N} 2$ & C9 & $\mathrm{C} 32$ & $-174.45(11)$ \\
\hline C9 & $\mathrm{N} 2$ & C6 & $\mathrm{C} 1$ & $178.36(11)$ & C9 & $\mathrm{N} 2$ & C6 & $\mathrm{C} 7$ & $-1.40(14)$ \\
\hline C6 & $\mathrm{N} 2$ & B4 & $\mathrm{F} 1$ & $142.05(12)$ & C6 & $\mathrm{N} 2$ & B4 & $\mathrm{F} 2$ & $-95.62(14)$ \\
\hline C6 & $\mathrm{N} 2$ & B4 & N1 & $21.98(16)$ & B4 & $\mathrm{N} 2$ & C6 & $\mathrm{C} 1$ & $-20.12(18)$ \\
\hline B4 & $\mathrm{N} 2$ & C6 & $\mathrm{C} 7$ & $160.11(10)$ & $\mathrm{C} 9$ & $\mathrm{~N} 2$ & B4 & $\mathrm{F} 1$ & $-59.57(18)$ \\
\hline C9 & $\mathrm{N} 2$ & B4 & $\mathrm{F} 2$ & $62.76(17)$ & C9 & $\mathrm{N} 2$ & B4 & N1 & $-179.64(11)$ \\
\hline B4 & $\mathrm{N} 2$ & C9 & $\mathrm{C} 8$ & $-158.22(11)$ & B4 & $\mathrm{N} 2$ & C9 & $\mathrm{C} 32$ & $25.2(2)$ \\
\hline $\mathrm{C} 2$ & $\mathrm{C} 1$ & C6 & $\mathrm{N} 2$ & $4.5(2)$ & $\mathrm{C} 2$ & $\mathrm{C} 1$ & C6 & $\mathrm{C} 7$ & $-175.79(12)$ \\
\hline C6 & $\mathrm{C} 1$ & $\mathrm{C} 2$ & N1 & $6.8(2)$ & C6 & $\mathrm{C} 1$ & $\mathrm{C} 2$ & $\mathrm{C} 3$ & $-172.35(12)$ \\
\hline N1 & $\mathrm{C} 2$ & $\mathrm{C} 3$ & $\mathrm{C} 4$ & $0.17(14)$ & N1 & $\mathrm{C} 2$ & $\mathrm{C} 3$ & $\mathrm{C} 10$ & $-178.32(12)$ \\
\hline $\mathrm{C} 1$ & $\mathrm{C} 2$ & $\mathrm{C} 3$ & $\mathrm{C} 4$ & $179.41(13)$ & $\mathrm{C} 1$ & $\mathrm{C} 2$ & $\mathrm{C} 3$ & $\mathrm{C} 10$ & $0.9(3)$ \\
\hline $\mathrm{C} 2$ & $\mathrm{C} 3$ & $\mathrm{C} 4$ & $\mathrm{C} 5$ & $0.43(14)$ & $\mathrm{C} 2$ & $\mathrm{C} 3$ & $\mathrm{C} 4$ & $\mathrm{C} 13$ & $176.51(11)$ \\
\hline $\mathrm{C} 2$ & $\mathrm{C} 3$ & $\mathrm{C} 10$ & $\mathrm{C} 11$ & $-179.56(13)$ & $\mathrm{C} 4$ & $\mathrm{C} 3$ & $\mathrm{C} 10$ & $\mathrm{C} 11$ & $2.12(19)$ \\
\hline $\mathrm{C} 10$ & $\mathrm{C} 3$ & $\mathrm{C} 4$ & $\mathrm{C} 5$ & $179.15(11)$ & $\mathrm{C} 10$ & $\mathrm{C} 3$ & $\mathrm{C} 4$ & $\mathrm{C} 13$ & $-4.78(19)$ \\
\hline $\mathrm{C} 3$ & $\mathrm{C} 4$ & $\mathrm{C} 5$ & N1 & $-0.90(14)$ & $\mathrm{C} 3$ & $\mathrm{C} 4$ & $\mathrm{C} 5$ & $\mathrm{C} 26$ & $175.64(11)$ \\
\hline $\mathrm{C} 3$ & $\mathrm{C} 4$ & $\mathrm{C} 13$ & $\mathrm{C} 12$ & $3.07(19)$ & $\mathrm{C} 5$ & $\mathrm{C} 4$ & $\mathrm{C} 13$ & $\mathrm{C} 12$ & $178.01(13)$ \\
\hline $\mathrm{C} 13$ & $\mathrm{C} 4$ & $\mathrm{C} 5$ & N1 & $-176.33(13)$ & $\mathrm{C} 13$ & $\mathrm{C} 4$ & $\mathrm{C} 5$ & $\mathrm{C} 26$ & $0.2(2)$ \\
\hline N1 & $\mathrm{C} 5$ & $\mathrm{C} 26$ & $\mathrm{C} 27$ & $-142.11(12)$ & $\mathrm{N} 1$ & $\mathrm{C} 5$ & $\mathrm{C} 26$ & $\mathrm{C} 31$ & $42.46(19)$ \\
\hline $\mathrm{C} 4$ & $\mathrm{C} 5$ & $\mathrm{C} 26$ & $\mathrm{C} 27$ & $41.96(19)$ & $\mathrm{C} 4$ & $\mathrm{C} 5$ & $\mathrm{C} 26$ & $\mathrm{C} 31$ & $-133.46(13)$ \\
\hline N2 & C6 & $\mathrm{C} 7$ & $\mathrm{C} 8$ & $0.11(14)$ & $\mathrm{N} 2$ & C6 & $\mathrm{C} 7$ & $\mathrm{C} 14$ & $-177.84(12)$ \\
\hline $\mathrm{C} 1$ & C6 & $\mathrm{C} 7$ & $\mathrm{C} 8$ & $-179.63(13)$ & $\mathrm{C} 1$ & C6 & $\mathrm{C} 7$ & $\mathrm{C} 14$ & $2.4(3)$ \\
\hline C6 & $\mathrm{C} 7$ & $\mathrm{C} 8$ & $\mathrm{C} 9$ & $1.13(14)$ & C6 & $\mathrm{C} 7$ & $\mathrm{C} 8$ & $\mathrm{C} 17$ & $-179.94(11)$ \\
\hline $\mathrm{C} 6$ & $\mathrm{C} 7$ & $\mathrm{C} 14$ & $\mathrm{C} 15$ & $176.24(13)$ & $\mathrm{C} 8$ & $\mathrm{C} 7$ & $\mathrm{C} 14$ & $\mathrm{C} 15$ & $-1.48(19)$ \\
\hline $\mathrm{C} 14$ & $\mathrm{C} 7$ & $\mathrm{C} 8$ & $\mathrm{C} 9$ & $179.38(11)$ & $\mathrm{C} 14$ & $\mathrm{C} 7$ & $\mathrm{C} 8$ & $\mathrm{C} 17$ & $-1.69(19)$ \\
\hline $\mathrm{C} 7$ & $\mathrm{C} 8$ & C9 & $\mathrm{N} 2$ & $-2.02(14)$ & $\mathrm{C} 7$ & $\mathrm{C} 8$ & $\mathrm{C} 9$ & $\mathrm{C} 32$ & $174.56(11)$ \\
\hline $\mathrm{C} 7$ & $\mathrm{C} 8$ & $\mathrm{C} 17$ & $\mathrm{C} 16$ & $3.00(19)$ & C9 & $\mathrm{C} 8$ & $\mathrm{C} 17$ & $\mathrm{C} 16$ & $-178.38(13)$ \\
\hline $\mathrm{C} 17$ & $\mathrm{C} 8$ & $\mathrm{C} 9$ & $\mathrm{~N} 2$ & $179.22(13)$ & $\mathrm{C} 17$ & $\mathrm{C} 8$ & $\mathrm{C} 9$ & $\mathrm{C} 32$ & $-4.2(2)$ \\
\hline $\mathrm{N} 2$ & C9 & $\mathrm{C} 32$ & $\mathrm{C} 33$ & 48.1(2) & $\mathrm{N} 2$ & C9 & $\mathrm{C} 32$ & $\mathrm{C} 37$ & $-135.93(13)$ \\
\hline $\mathrm{C} 8$ & C9 & $\mathrm{C} 32$ & $\mathrm{C} 33$ & $-127.90(14)$ & $\mathrm{C} 8$ & $\mathrm{C} 9$ & $\mathrm{C} 32$ & $\mathrm{C} 37$ & $48.05(19)$ \\
\hline $\mathrm{C} 3$ & $\mathrm{C} 10$ & $\mathrm{C} 11$ & $\mathrm{C} 12$ & $2.0(2)$ & $\mathrm{C} 3$ & $\mathrm{C} 10$ & $\mathrm{C} 11$ & $\mathrm{C} 18$ & $-178.73(11)$ \\
\hline $\mathrm{C} 10$ & $\mathrm{C} 11$ & $\mathrm{C} 12$ & $\mathrm{C} 13$ & $-3.7(2)$ & $\mathrm{C} 10$ & $\mathrm{C} 11$ & $\mathrm{C} 12$ & $\mathrm{C} 21$ & $175.69(11)$ \\
\hline $\mathrm{C} 10$ & $\mathrm{C} 11$ & $\mathrm{C} 18$ & C19 & $-176.70(12)$ & $\mathrm{C} 12$ & $\mathrm{C} 11$ & $\mathrm{C} 18$ & C19 & $2.6(2)$ \\
\hline $\mathrm{C} 18$ & $\mathrm{C} 11$ & $\mathrm{C} 12$ & $\mathrm{C} 13$ & $177.05(12)$ & $\mathrm{C} 18$ & $\mathrm{C} 11$ & $\mathrm{C} 12$ & $\mathrm{C} 21$ & $-3.59(19)$ \\
\hline $\mathrm{C} 11$ & $\mathrm{C} 12$ & $\mathrm{C} 13$ & $\mathrm{C} 4$ & $1.05(19)$ & $\mathrm{C} 11$ & $\mathrm{C} 12$ & $\mathrm{C} 21$ & $\mathrm{C} 20$ & $2.3(2)$ \\
\hline $\mathrm{C} 13$ & $\mathrm{C} 12$ & $\mathrm{C} 21$ & $\mathrm{C} 20$ & $-178.34(12)$ & $\mathrm{C} 21$ & $\mathrm{C} 12$ & $\mathrm{C} 13$ & $\mathrm{C} 4$ & $-178.29(11)$ \\
\hline $\mathrm{C} 7$ & $\mathrm{C} 14$ & $\mathrm{C} 15$ & $\mathrm{C} 16$ & $3.2(2)$ & $\mathrm{C} 7$ & $\mathrm{C} 14$ & $\mathrm{C} 15$ & $\mathrm{C} 22$ & $-173.80(11)$ \\
\hline $\mathrm{C} 14$ & $\mathrm{C} 15$ & $\mathrm{C} 16$ & $\mathrm{C} 17$ & $-1.90(19)$ & $\mathrm{C} 14$ & $\mathrm{C} 15$ & $\mathrm{C} 16$ & $\mathrm{C} 25$ & $179.76(11)$ \\
\hline $\mathrm{C} 14$ & $\mathrm{C} 15$ & $\mathrm{C} 22$ & $\mathrm{C} 23$ & $177.97(12)$ & $\mathrm{C} 16$ & $\mathrm{C} 15$ & $\mathrm{C} 22$ & $\mathrm{C} 23$ & $0.9(2)$ \\
\hline $\mathrm{C} 22$ & $\mathrm{C} 15$ & $\mathrm{C} 16$ & $\mathrm{C} 17$ & $175.20(12)$ & $\mathrm{C} 22$ & $\mathrm{C} 15$ & $\mathrm{C} 16$ & $\mathrm{C} 25$ & $-3.13(19)$ \\
\hline $\mathrm{C} 15$ & $\mathrm{C} 16$ & $\mathrm{C} 17$ & $\mathrm{C} 8$ & $-1.21(19)$ & $\mathrm{C} 15$ & $\mathrm{C} 16$ & $\mathrm{C} 25$ & $\mathrm{C} 24$ & $3.0(2)$ \\
\hline $\mathrm{C} 17$ & $\mathrm{C} 16$ & $\mathrm{C} 25$ & $\mathrm{C} 24$ & $-175.36(12)$ & $\mathrm{C} 25$ & $\mathrm{C} 16$ & $\mathrm{C} 17$ & $\mathrm{C} 8$ & $177.07(11)$ \\
\hline $\mathrm{C} 11$ & $\mathrm{C} 18$ & $\mathrm{C} 19$ & $\mathrm{C} 20$ & $-0.1(2)$ & $\mathrm{C} 18$ & $\mathrm{C} 19$ & $\mathrm{C} 20$ & $\mathrm{C} 21$ & $-1.3(2)$ \\
\hline $\mathrm{C} 19$ & $\mathrm{C} 20$ & $\mathrm{C} 21$ & $\mathrm{C} 12$ & $0.1(2)$ & $\mathrm{C} 15$ & $\mathrm{C} 22$ & $\mathrm{C} 23$ & $\mathrm{C} 24$ & $1.6(2)$ \\
\hline $\mathrm{C} 22$ & $\mathrm{C} 23$ & $\mathrm{C} 24$ & $\mathrm{C} 25$ & $-1.9(2)$ & $\mathrm{C} 23$ & $\mathrm{C} 24$ & $\mathrm{C} 25$ & $\mathrm{C} 16$ & $-0.5(2)$ \\
\hline $\mathrm{C} 5$ & $\mathrm{C} 26$ & $\mathrm{C} 27$ & $\mathrm{C} 28$ & $-174.95(11)$ & $\mathrm{C} 5$ & $\mathrm{C} 26$ & $\mathrm{C} 31$ & $\mathrm{C} 30$ & $175.39(11)$ \\
\hline $\mathrm{C} 27$ & $\mathrm{C} 26$ & $\mathrm{C} 31$ & $\mathrm{C} 30$ & $-0.1(2)$ & $\mathrm{C} 31$ & $\mathrm{C} 26$ & $\mathrm{C} 27$ & $\mathrm{C} 28$ & $0.65(19)$ \\
\hline $\mathrm{C} 26$ & $\mathrm{C} 27$ & $\mathrm{C} 28$ & $\mathrm{C} 29$ & $-0.7(2)$ & $\mathrm{C} 27$ & $\mathrm{C} 28$ & $\mathrm{C} 29$ & $\mathrm{C} 30$ & $0.2(2)$ \\
\hline $\mathrm{C} 28$ & $\mathrm{C} 29$ & $\mathrm{C} 30$ & $\mathrm{C} 31$ & $0.4(2)$ & $\mathrm{C} 29$ & $\mathrm{C} 30$ & $\mathrm{C} 31$ & $\mathrm{C} 26$ & $-0.5(2)$ \\
\hline $\mathrm{C} 9$ & $\mathrm{C} 32$ & $\mathrm{C} 33$ & $\mathrm{C} 34$ & $176.70(12)$ & C9 & $\mathrm{C} 32$ & $\mathrm{C} 37$ & $\mathrm{C} 36$ & $-177.39(12)$ \\
\hline $\mathrm{C} 33$ & $\mathrm{C} 32$ & $\mathrm{C} 37$ & $\mathrm{C} 36$ & $-1.3(2)$ & $\mathrm{C} 37$ & $\mathrm{C} 32$ & $\mathrm{C} 33$ & C34 & $0.7(2)$ \\
\hline $\mathrm{C} 32$ & $\mathrm{C} 33$ & $\mathrm{C} 34$ & C35 & $-0.0(2)$ & $\mathrm{C} 33$ & $\mathrm{C} 34$ & $\mathrm{C} 35$ & $\mathrm{C} 36$ & $-0.0(2)$ \\
\hline $\mathrm{C} 34$ & $\mathrm{C} 35$ & $\mathrm{C} 36$ & C37 & $-0.6(2)$ & $\mathrm{C} 35$ & $\mathrm{C} 36$ & $\mathrm{C} 37$ & $\mathrm{C} 32$ & $1.3(2)$ \\
\hline
\end{tabular}


Table S4. Atom coordinates and absolute energy levels for $\mathbf{7}$ optimized in the $\mathrm{S}_{0}$ state.

\begin{tabular}{|c|c|c|c|c|c|}
\hline \multirow{2}{*}{$\begin{array}{l}\text { Center } \\
\text { Number }\end{array}$} & \multirow{2}{*}{$\begin{array}{l}\text { Atomic } \\
\text { Number }\end{array}$} & \multirow{2}{*}{$\begin{array}{c}\text { Atomic } \\
\text { Type }\end{array}$} & \multicolumn{3}{|c|}{ Coordinates (Angstroms) } \\
\hline & & & $x$ & Y & Z \\
\hline 1 & 6 & 0 & 1.216242 & -1.807875 & 0.082314 \\
\hline 2 & 7 & 0 & 1.280773 & -0.412826 & 0.095193 \\
\hline 3 & 7 & 0 & -1.280727 & -0.412820 & -0.094594 \\
\hline 4 & 6 & 0 & -1.216139 & -1.807834 & -0.081581 \\
\hline 5 & 6 & 0 & 0.000049 & -2.468493 & 0.000417 \\
\hline 6 & 6 & 0 & 2.551607 & -2.317128 & 0.099030 \\
\hline 7 & 6 & 0 & 3.411874 & -1.183585 & 0.096591 \\
\hline 8 & 6 & 0 & 2.575867 & -0.016611 & 0.083490 \\
\hline 9 & 6 & 0 & -2.575880 & -0.016645 & -0.083460 \\
\hline 10 & 6 & 0 & -3.411796 & -1.183668 & -0.096730 \\
\hline 11 & 6 & 0 & -2.551472 & -2.317168 & -0.098668 \\
\hline 12 & 6 & 0 & 3.085944 & -3.616794 & 0.122831 \\
\hline 13 & 6 & 0 & 4.463243 & -3.761783 & 0.157782 \\
\hline 14 & 6 & 0 & 5.320821 & -2.636356 & 0.179138 \\
\hline 15 & 6 & 0 & 4.809980 & -1.350213 & 0.153500 \\
\hline 16 & 6 & 0 & -4.809878 & -1.350399 & -0.154229 \\
\hline 17 & 6 & 0 & -5.320625 & -2.636572 & -0.179921 \\
\hline 18 & 6 & 0 & -4.462989 & -3.761953 & -0.158036 \\
\hline 19 & 6 & 0 & -3.085720 & -3.616873 & -0.122511 \\
\hline 20 & 6 & 0 & 3.062640 & 1.372048 & 0.042003 \\
\hline 21 & 6 & 0 & -3.062734 & 1.371987 & -0.042034 \\
\hline 22 & 6 & 0 & 2.591914 & 2.371893 & 0.911111 \\
\hline 23 & 6 & 0 & 3.135502 & 3.653646 & 0.866189 \\
\hline 24 & 6 & 0 & 4.149913 & 3.964625 & -0.040674 \\
\hline 25 & 6 & 0 & 4.622200 & 2.980947 & -0.910966 \\
\hline 26 & 6 & 0 & 4.088627 & 1.695309 & -0.866925 \\
\hline 27 & 6 & 0 & -4.089078 & 1.695054 & 0.866579 \\
\hline 28 & 6 & 0 & -4.622748 & 2.980645 & 0.910637 \\
\hline 29 & 6 & 0 & -4.150202 & 3.964502 & 0.040682 \\
\hline 30 & 6 & 0 & -3.135435 & 3.653731 & -0.865845 \\
\hline 31 & 6 & 0 & -2.591752 & 2.372016 & -0.910785 \\
\hline 32 & 5 & 0 & -0.000043 & 0.517811 & 0.000054 \\
\hline 33 & 9 & 0 & -0.128474 & 1.282879 & 1.146919 \\
\hline 34 & 9 & 0 & 0.128504 & 1.282233 & -1.147209 \\
\hline 35 & 1 & 0 & 0.000008 & -3.553479 & 0.000471 \\
\hline 36 & 1 & 0 & 2.439326 & -4.489507 & 0.120928 \\
\hline 37 & 1 & 0 & 4.896593 & -4.757426 & 0.178749 \\
\hline 38 & 1 & 0 & 6.394841 & -2.788769 & 0.222724 \\
\hline 39 & 1 & 0 & 5.469489 & -0.489553 & 0.185324 \\
\hline 40 & 1 & 0 & -5.469436 & -0.489791 & -0.186435 \\
\hline 41 & 1 & 0 & -6.394618 & -2.789057 & -0.223956 \\
\hline 42 & 1 & 0 & -4.896277 & -4.757622 & -0.179056 \\
\hline 43 & 1 & 0 & -2.439040 & -4.489539 & -0.120196 \\
\hline 44 & 1 & 0 & 1.800165 & 2.141114 & 1.610456 \\
\hline 45 & 1 & 0 & 2.763199 & 4.413454 & 1.546956 \\
\hline 46 & 1 & 0 & 4.567490 & 4.966762 & -0.071257 \\
\hline 47 & 1 & 0 & 5.402676 & 3.214749 & -1.629082 \\
\hline 48 & 1 & 0 & 4.444375 & 0.939411 & -1.559277 \\
\hline 49 & 1 & 0 & -4.445021 & 0.939031 & 1.558694 \\
\hline 50 & 1 & 0 & -5.403504 & 3.214278 & 1.628506 \\
\hline 51 & 1 & 0 & -4.567851 & 4.966609 & 0.071283 \\
\hline 52 & 1 & 0 & -2.762909 & 4.413667 & -1.546348 \\
\hline 53 & 1 & 0 & -1.799744 & 2.141420 & -1.609897 \\
\hline
\end{tabular}


Table S5. Atom coordinates and absolute energy levels for 1a optimized in the $\mathrm{S}_{0}$ state.

\begin{tabular}{|c|c|c|c|c|c|}
\hline \multirow{2}{*}{$\begin{array}{l}\text { Center } \\
\text { Number }\end{array}$} & \multirow{2}{*}{$\begin{array}{l}\text { Atomic } \\
\text { Number }\end{array}$} & \multirow{2}{*}{$\begin{array}{c}\text { Atomic } \\
\text { Type }\end{array}$} & \multicolumn{3}{|c|}{ Coordinates（Angstroms） } \\
\hline & & & $x$ & $\mathrm{Y}$ & Z \\
\hline 1 & 6 & 0 & 1.218169 & -1.008698 & 0.061047 \\
\hline 2 & 7 & 0 & 1.285043 & 0.386611 & 0.072125 \\
\hline 3 & 7 & 0 & -1.285051 & 0.386635 & -0.071800 \\
\hline 4 & 6 & 0 & -1.218144 & -1.008665 & -0.060652 \\
\hline 5 & 6 & 0 & 0.000006 & -1.667460 & 0.000222 \\
\hline 6 & 6 & 0 & 2.557798 & -1.518482 & 0.056802 \\
\hline 7 & 6 & 0 & 3.420511 & -0.370025 & 0.039936 \\
\hline 8 & 6 & 0 & 2.574369 & 0.793880 & 0.039228 \\
\hline 9 & 6 & 0 & -2.574412 & 0.793874 & -0.039158 \\
\hline 10 & 6 & 0 & -3.420504 & -0.370052 & -0.039895 \\
\hline 11 & 6 & 0 & -2.557759 & -1.518484 & -0.056541 \\
\hline 12 & 6 & 0 & 3.088475 & -2.799842 & 0.073125 \\
\hline 13 & 6 & 0 & 4.487424 & -2.970880 & 0.086040 \\
\hline 14 & 6 & 0 & 5.357482 & -1.813218 & 0.093815 \\
\hline 15 & 6 & 0 & 4.802612 & -0.519824 & 0.075284 \\
\hline 16 & 6 & 0 & 5.084469 & -4.267108 & 0.103564 \\
\hline 17 & 6 & 0 & 6.447313 & -4.420858 & 0.129347 \\
\hline 18 & 6 & 0 & 7.302306 & -3.283322 & 0.140676 \\
\hline 19 & 6 & 0 & 6.770036 & -2.019848 & 0.124022 \\
\hline 20 & 6 & 0 & -4.802603 & -0.519889 & -0.075444 \\
\hline 21 & 6 & 0 & -5.357429 & -1.813295 & -0.093983 \\
\hline 22 & 6 & 0 & -4.487340 & -2.970935 & -0.085987 \\
\hline 23 & 6 & 0 & -3.088400 & -2.799862 & -0.072848 \\
\hline 24 & 6 & 0 & -6.769976 & -2.019970 & -0.124418 \\
\hline 25 & 6 & 0 & -7.302206 & -3.283458 & -0.141086 \\
\hline 26 & 6 & 0 & -6.447181 & -4.420972 & -0.129542 \\
\hline 27 & 6 & 0 & -5.084348 & -4.267182 & -0.103535 \\
\hline 28 & 6 & 0 & 3.053025 & 2.184518 & -0.012394 \\
\hline 29 & 6 & 0 & -3.053098 & 2.184507 & 0.012373 \\
\hline 30 & 6 & 0 & 2.597051 & 3.182742 & 0.866704 \\
\hline 31 & 6 & 0 & 3.133916 & 4.466798 & 0.809969 \\
\hline 32 & 6 & 0 & 4.127499 & 4.782104 & -0.118243 \\
\hline 33 & 6 & 0 & 4.585558 & 3.800139 & -0.998048 \\
\hline 34 & 6 & 0 & 4.058617 & 2.512257 & -0.942736 \\
\hline 35 & 6 & 0 & -4.058777 & 2.512250 & 0.942621 \\
\hline 36 & 6 & 0 & -4.585738 & 3.800124 & 0.997868 \\
\hline 37 & 6 & 0 & -4.127610 & 4.782083 & 0.118088 \\
\hline 38 & 6 & 0 & -3.133940 & 4.466776 & -0.810027 \\
\hline 39 & 6 & 0 & -2.597054 & 3.182724 & -0.866694 \\
\hline 40 & 5 & 0 & -0.000053 & 1.315927 & 0.000098 \\
\hline 41 & 9 & 0 & -0.108033 & 2.078371 & 1.149864 \\
\hline 42 & 9 & 0 & 0.107984 & 2.078243 & -1.149694 \\
\hline 43 & 1 & 0 & -0.000024 & -2.752336 & 0.000244 \\
\hline 44 & 1 & 0 & 2.447394 & -3.677445 & 0.084437 \\
\hline 45 & 1 & 0 & 5.458490 & 0.344864 & 0.096406 \\
\hline 46 & 1 & 0 & 4.433068 & -5.137145 & 0.096871 \\
\hline 47 & 1 & 0 & 6.881798 & -5.416104 & 0.142267 \\
\hline 48 & 1 & 0 & 8.378942 & -3.422346 & 0.162574 \\
\hline 49 & 1 & 0 & 7.419103 & -1.148058 & 0.132552 \\
\hline 50 & 1 & 0 & -5.458498 & 0.344781 & -0.096725 \\
\hline 51 & 1 & 0 & -2.447296 & -3.677451 & -0.083986 \\
\hline 52 & 1 & 0 & -7.419065 & -1.148198 & -0.133110 \\
\hline 53 & 1 & 0 & -8.378833 & -3.422517 & -0.163158 \\
\hline 54 & 1 & 0 & -6.881639 & -5.416230 & -0.142474 \\
\hline 55 & 1 & 0 & -4.432922 & -5.137200 & -0.096678 \\
\hline 56 & 1 & 0 & 1.822479 & 2.948580 & 1.583813 \\
\hline 57 & 1 & 0 & 2.773197 & 5.224917 & 1.498808 \\
\hline 58 & 1 & 0 & 4.539995 & 5.786017 & -0.157753 \\
\hline 59 & 1 & 0 & 5.349541 & 4.037142 & -1.732676 \\
\hline 60 & 1 & 0 & 4.402881 & 1.757795 & -1.642382 \\
\hline 61 & 1 & 0 & -4.403092 & 1.757791 & 1.642246 \\
\hline 62 & 1 & 0 & -5.349788 & 4.037134 & 1.732423 \\
\hline 63 & 1 & 0 & -4.540121 & 5.785992 & 0.157553 \\
\hline 64 & 1 & 0 & -2.773164 & 5.224888 & -1.498844 \\
\hline 65 & 1 & 0 & -1.822414 & 2.948566 & -1.583731 \\
\hline
\end{tabular}


Table S6. Atom coordinates and absolute energy levels for $\mathbf{1 b}$ optimized in the $\mathrm{S}_{0}$ state.

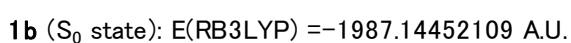

\begin{tabular}{|c|c|c|c|c|c|}
\hline \multirow{2}{*}{$\begin{array}{l}\text { Center } \\
\text { Number }\end{array}$} & \multirow{2}{*}{$\begin{array}{l}\text { Atomic } \\
\text { Number }\end{array}$} & \multirow{2}{*}{$\begin{array}{c}\text { Atomic } \\
\text { Type }\end{array}$} & \multicolumn{3}{|c|}{ Coordinates (Angstroms) } \\
\hline & & & $\mathrm{x}$ & Y & Z \\
\hline 1 & 6 & 0 & -1.219075 & 1.339691 & -0.015901 \\
\hline 2 & 7 & 0 & -1.276073 & -0.056583 & 0.024209 \\
\hline 3 & 7 & 0 & 1.276081 & -0.056537 & -0.025526 \\
\hline 4 & 6 & 0 & 1.218985 & 1.339737 & 0.014353 \\
\hline 5 & 6 & 0 & -0.000066 & 2.001702 & -0.000842 \\
\hline 6 & 6 & 0 & -2.565315 & 1.831451 & -0.092169 \\
\hline 7 & 6 & 0 & -3.412418 & 0.669067 & -0.102767 \\
\hline 8 & 6 & 0 & -2.553997 & -0.473414 & -0.020459 \\
\hline 9 & 6 & 0 & 2.554047 & -0.473274 & 0.019975 \\
\hline 10 & 6 & 0 & 3.412326 & 0.669277 & 0.102498 \\
\hline 11 & 6 & 0 & 2.565140 & 1.831604 & 0.091195 \\
\hline 12 & 6 & 0 & -3.122427 & 3.100181 & -0.155155 \\
\hline 13 & 6 & 0 & -4.523989 & 3.239805 & -0.229221 \\
\hline 14 & 6 & 0 & -5.373808 & 2.065941 & -0.235413 \\
\hline 15 & 6 & 0 & -4.795207 & 0.785001 & -0.167779 \\
\hline 16 & 6 & 0 & -5.144370 & 4.523315 & -0.298726 \\
\hline 17 & 6 & 0 & -6.508385 & 4.651324 & -0.370064 \\
\hline 18 & 6 & 0 & -7.342514 & 3.498430 & -0.375684 \\
\hline 19 & 6 & 0 & -6.788293 & 2.245998 & -0.309902 \\
\hline 20 & 6 & 0 & 4.795081 & 0.785357 & 0.168174 \\
\hline 21 & 6 & 0 & 5.373544 & 2.066349 & 0.235835 \\
\hline 22 & 6 & 0 & 4.523632 & 3.240153 & 0.228914 \\
\hline 23 & 6 & 0 & 3.122131 & 3.100396 & 0.154133 \\
\hline 24 & 6 & 0 & 6.787977 & 2.246539 & 0.311028 \\
\hline 25 & 6 & 0 & 7.342066 & 3.499028 & 0.376799 \\
\hline 26 & 6 & 0 & 6.507849 & 4.651858 & 0.370453 \\
\hline 27 & 6 & 0 & 5.143883 & 4.523728 & 0.298425 \\
\hline 28 & 6 & 0 & -2.971917 & -1.889783 & 0.008666 \\
\hline 29 & 6 & 0 & 2.972144 & -1.889574 & -0.008708 \\
\hline 30 & 6 & 0 & -2.877995 & -2.639173 & 1.201283 \\
\hline 31 & 6 & 0 & -3.235068 & -3.992075 & 1.202902 \\
\hline 32 & 6 & 0 & -3.721645 & -4.585223 & 0.037118 \\
\hline 33 & 6 & 0 & -3.860604 & -3.843754 & -1.134571 \\
\hline 34 & 6 & 0 & -3.484702 & -2.500295 & -1.140492 \\
\hline 35 & 6 & 0 & 3.485393 & -2.499469 & 1.140579 \\
\hline 36 & 6 & 0 & 3.861534 & -3.842863 & 1.135178 \\
\hline 37 & 6 & 0 & 3.722359 & -4.584905 & -0.036119 \\
\hline 38 & 6 & 0 & 3.235364 & -3.992375 & -1.202048 \\
\hline 39 & 6 & 0 & 2.878078 & -2.639536 & -1.200970 \\
\hline 40 & 5 & 0 & 0.000131 & -1.002620 & -0.000432 \\
\hline 41 & 9 & 0 & 0.061708 & -1.771669 & 1.146254 \\
\hline 42 & 9 & 0 & -0.061515 & -1.772392 & -1.146269 \\
\hline 43 & 8 & 0 & -2.476542 & -1.955518 & 2.304469 \\
\hline 44 & 6 & 0 & -1.915026 & -2.689093 & 3.384750 \\
\hline 45 & 8 & 0 & 2.476302 & -1.956444 & -2.304393 \\
\hline 46 & 6 & 0 & 1.915041 & -2.690622 & -3.384388 \\
\hline 47 & 1 & 0 & -0.000089 & 3.086826 & -0.000861 \\
\hline 48 & 1 & 0 & -2.501958 & 3.992621 & -0.149180 \\
\hline 49 & 1 & 0 & -5.427507 & -0.098396 & -0.168165 \\
\hline 50 & 1 & 0 & -4.509355 & 5.405485 & -0.294255 \\
\hline 51 & 1 & 0 & -6.959658 & 5.637836 & -0.422462 \\
\hline 52 & 1 & 0 & -8.420422 & 3.616894 & -0.431929 \\
\hline 53 & 1 & 0 & -7.420939 & 1.362154 & -0.313455 \\
\hline 54 & 1 & 0 & 5.427456 & -0.097987 & 0.169052 \\
\hline 55 & 1 & 0 & 2.501588 & 3.992782 & 0.147569 \\
\hline 56 & 1 & 0 & 7.420692 & 1.362747 & 0.315132 \\
\hline 57 & 1 & 0 & 8.419935 & 3.617588 & 0.433589 \\
\hline 58 & 1 & 0 & 6.959014 & 5.638421 & 0.422843 \\
\hline 59 & 1 & 0 & 4.508806 & 5.405851 & 0.293402 \\
\hline 60 & 1 & 0 & -3.148149 & -4.578053 & 2.110266 \\
\hline 61 & 1 & 0 & -3.999442 & -5.635292 & 0.053568 \\
\hline 62 & 1 & 0 & -4.242770 & -4.306846 & -2.038527 \\
\hline 63 & 1 & 0 & -3.557272 & -1.916304 & -2.052924 \\
\hline 64 & 1 & 0 & 3.558115 & -1.915048 & 2.052724 \\
\hline 65 & 1 & 0 & 4.244043 & -4.305452 & 2.039247 \\
\hline 66 & 1 & 0 & 4.000312 & -5.634938 & -0.052163 \\
\hline
\end{tabular}


Table S7. Atom coordinates and absolute energy levels for $\mathbf{2}$ optimized in the $\mathrm{S}_{0}$ state.

\begin{tabular}{|c|c|c|c|c|c|}
\hline \multirow{2}{*}{$\begin{array}{l}\text { Center } \\
\text { Number }\end{array}$} & \multirow{2}{*}{$\begin{array}{l}\text { Atomic } \\
\text { Number }\end{array}$} & \multirow{2}{*}{$\begin{array}{l}\text { Atomic } \\
\text { Type }\end{array}$} & \multicolumn{3}{|c|}{ Coordinates (Angstroms) } \\
\hline & & & $x$ & $Y$ & Z \\
\hline 1 & 6 & 0 & -1.195190 & -1.069510 & 0.226608 \\
\hline 2 & 7 & 0 & -1.217791 & 0.320003 & 0.169986 \\
\hline 3 & 7 & 0 & 1.217628 & 0.319863 & -0.169342 \\
\hline 4 & 6 & 0 & 1.194890 & -1.069768 & -0.225536 \\
\hline 5 & 6 & 0 & -0.000118 & -1.749820 & 0.000640 \\
\hline 6 & 6 & 0 & -2.568952 & -1.494642 & 0.290113 \\
\hline 7 & 6 & 0 & -3.383946 & -0.305928 & 0.164682 \\
\hline 8 & 6 & 0 & -2.469465 & 0.805031 & 0.038160 \\
\hline 9 & 6 & 0 & 2.469290 & 0.804832 & -0.038200 \\
\hline 10 & 6 & 0 & 3.383747 & -0.306218 & -0.164922 \\
\hline 11 & 6 & 0 & 2.568697 & -1.494915 & -0.289613 \\
\hline 12 & 6 & 0 & -3.159485 & -2.743338 & 0.399684 \\
\hline 13 & 6 & 0 & -4.565093 & -2.855115 & 0.392242 \\
\hline 14 & 6 & 0 & -5.380754 & -1.669510 & 0.250864 \\
\hline 15 & 6 & 0 & -4.767760 & -0.405830 & 0.130659 \\
\hline 16 & 6 & 0 & -5.218131 & -4.117509 & 0.515118 \\
\hline 17 & 6 & 0 & -6.586830 & -4.213039 & 0.497383 \\
\hline 18 & 6 & 0 & -7.389063 & -3.047224 & 0.355110 \\
\hline 19 & 6 & 0 & -6.800103 & -1.814072 & 0.235605 \\
\hline 20 & 6 & 0 & 4.767545 & -0.406080 & -0.131390 \\
\hline 21 & 6 & 0 & 5.380494 & -1.669838 & -0.251488 \\
\hline 22 & 6 & 0 & 4.564769 & -2.855456 & -0.392188 \\
\hline 23 & 6 & 0 & 3.159121 & -2.743650 & -0.399048 \\
\hline 24 & 6 & 0 & 6.799825 & -1.814396 & -0.236726 \\
\hline 25 & 6 & 0 & 7.388736 & -3.047610 & -0.356081 \\
\hline 26 & 6 & 0 & 6.586445 & -4.213428 & -0.497694 \\
\hline 27 & 6 & 0 & 5.217721 & -4.117879 & -0.514938 \\
\hline 28 & 6 & 0 & -2.611104 & 2.160134 & -0.444892 \\
\hline 29 & 6 & 0 & 2.611273 & 2.159921 & 0.444757 \\
\hline 30 & 6 & 0 & -3.816194 & 2.888824 & -0.421786 \\
\hline 31 & 6 & 0 & -3.926057 & 4.109771 & -1.071114 \\
\hline 32 & 6 & 0 & -2.827453 & 4.622825 & -1.775143 \\
\hline 33 & 6 & 0 & -1.619543 & 3.935473 & -1.802422 \\
\hline 34 & 6 & 0 & -1.482550 & 2.718512 & -1.123703 \\
\hline 35 & 6 & 0 & 1.482990 & 2.718323 & 1.124124 \\
\hline 36 & 6 & 0 & 1.620427 & 3.935178 & 1.802951 \\
\hline 37 & 6 & 0 & 2.828339 & 4.622496 & 1.775052 \\
\hline 38 & 6 & 0 & 3.926551 & 4.109515 & 1.070347 \\
\hline 39 & 6 & 0 & 3.816339 & 2.888604 & 0.421021 \\
\hline 40 & 5 & 0 & 0.000075 & 1.232846 & 0.000393 \\
\hline 41 & 8 & 0 & -0.289786 & 2.087484 & -1.156263 \\
\hline 42 & 8 & 0 & 0.290260 & 2.087318 & 1.157184 \\
\hline 43 & 1 & 0 & -0.000200 & -2.834203 & 0.000831 \\
\hline 44 & 1 & 0 & -2.556893 & -3.642832 & 0.494606 \\
\hline 45 & 1 & 0 & -5.395273 & 0.470107 & 0.006115 \\
\hline 46 & 1 & 0 & -4.605821 & -5.008965 & 0.623073 \\
\hline 47 & 1 & 0 & -7.065493 & -5.183283 & 0.591794 \\
\hline 48 & 1 & 0 & -8.470877 & -3.139215 & 0.342014 \\
\hline 49 & 1 & 0 & -7.409772 & -0.920706 & 0.127814 \\
\hline 50 & 1 & 0 & 5.395134 & 0.469875 & -0.007364 \\
\hline 51 & 1 & 0 & 2.556477 & -3.643161 & -0.493425 \\
\hline 52 & 1 & 0 & 7.409564 & -0.921016 & -0.129466 \\
\hline 53 & 1 & 0 & 8.470555 & -3.139593 & -0.343374 \\
\hline 54 & 1 & 0 & 7.065034 & -5.183721 & -0.591994 \\
\hline 55 & 1 & 0 & 4.605379 & -5.009369 & -0.622417 \\
\hline 56 & 1 & 0 & -4.661373 & 2.494651 & 0.131918 \\
\hline 57 & 1 & 0 & -4.859278 & 4.662477 & -1.035102 \\
\hline 58 & 1 & 0 & -2.912161 & 5.574165 & -2.292280 \\
\hline 59 & 1 & 0 & -0.754478 & 4.327479 & -2.326447 \\
\hline 60 & 1 & 0 & 0.755674 & 4.327170 & 2.327503 \\
\hline 61 & 1 & 0 & 2.913354 & 5.573787 & 2.292232 \\
\hline 62 & 1 & 0 & 4.859742 & 4.662237 & 1.033865 \\
\hline 63 & 1 & 0 & 4.661216 & 2.494418 & -0.133150 \\
\hline
\end{tabular}

